\title{
Buckling and wrinkling of anisotropic sandwich plates
}

\author{
Riccardo Vescovini, Michele D’Ottavio, Lorenzo Dozio, Olivier Polit
}

\section{Introduction}

Sandwich panels are a particular case of composite structures, which consist of two thin and stiff layers, the facesheets, that are separated by a rather thick and weak layer, the core. The geometric and constitutive mismatch between the skins and the core provides the sandwich panel with high specific bending stiffness which, in turn, provides beneficial effects over the buckling response. For this reason, sandwich panels are often employed as structural elements in buckling critical regions, where compressive and shear loads may promote elastic instability phenomena. Hence it is not surprising that the first scientific paper dedicated to sandwich panels, written by Marguerre in 1944, addressed the buckling problem (Vinson, 1999). Space launchers and ship hulls are few but examples of buckling-driven designs where sandwich panels have been successfully employed. As opposed to the case of thin-walled panels, a complicating effect of sandwich panels is due to the strong mismatch of geometric and constitutive properties along the thickness. This feature induces sharp gradients across the thickness direction, even in the presence of a smooth response over the plate's major dimensions, i.e., global buckling or long wavelength response. In addition, and depending on the elastic and geometrical relative properties of core and faces, the buckling behavior can be characterized by a local response, commonly denoted as wrinkling-type instability, with halfwaves of the order of the panel thickness (Carlsson \& Kardomateas, 2011; Ley, Lin, \& Mbanefo, 1999). Both local and global instabilities are generally prevented from design operative conditions, as they may lead the structure to premature failure. For this reason, accurate yet reliable analysis tools are of crucial importance when designing a sandwich structure against buckling or, to a more general extent, by accounting for buckling requirements. While early sandwich designs made

\footnotetext{
* Corresponding author.

E-mail address: riccardo.vescovini@polimi.it (R. Vescovini).
} 
large use of isotropic and cross-ply facesheets, increasing attention has been dedicated in the years to fully exploit the tailoring potentialities offered by composites, thus enlarging the class of suitable configurations to include those characterized by anisotropic faces. This also demands for improved modeling capabilities, meaning that improved higher-order theories (Khdeir, Reddy, \& Frederick, 1989; Librescu \& Reddy, 1989; Piskunov, Verijenko, Adal, \& Summers, 1993; Reddy \& Liu, 1985), axiomatic approaches (Carrera, 2002; 2003a; 2003b; Carrera, Pagani, \& Valvano, 2017; Demasi, 2009a; 2009b; 2009c) as well as asymptotic approaches (Berdichevsky, 1979; 2010; Yu, Hodges, \& Volovoi, 2002) can be a useful framework for coping with the peculiar aspects offered by highly heterogeneous laminates. An interesting assessment between the capabilities offered by axiomatic and asymptotic strategies can be found in Demasi and Yu (2013). Among the theories specifically developed for analyzing sandwich structures, it is worth highlighting the high-order sandwich panel theory (HSAPT) due to Frostig, Baruch, Vilnay, and Sheinman (1992). It is based on Euler-Bernoulli beam theory for the skins, while transverse and in-plane displacements in the core are described using second and third order polynomials, respectively. The theory accounts for transverse and shear stresses in the core, but neglects the in-plane stresses in the core. A successful application of HSAPT to curved panels in the presence of debonding between core and faces is illustrated in Frostig and Thomsen (2011). An improvement to HSAPT is given by its enhanced version, commonly referred to as extended sandwich plate theory (EHSAPT) and due to Phan, Frostig, and Kardomateas (2012). The theory accounts for in-plane rigidity of the core and leads to a kinematic model where three generalized displacement coordinates are associated with the core instead of just one, as in HSAPT. The theory has been applied to analyze free vibration (Phan, Frostig, \& Kardomateas, 2013) and buckling problems (Phan, Bailey, Kardomateas, \& Battley, 2012; Phan, Kardomateas, \& Frostig, 2012), revealing good agreement with benchmark elasticity solutions due to Kardomateas $(2005,2010)$. Recently, a finite element formulation of EHSAPT was developed by Yuan, Kardomateas, and Frostig (2015). A comprehensive overview of analytical methods used for sandwich analysis is available in Birman and Kardomateas (2018).

In the context of global buckling evaluation, different strategies were developed in the past. Exact elasticity solution were derived for the case of sandwich struts (Ji \& Waas, 2012; Kardomateas, 2010), while other works focused on the obtainment of approximate solutions at panel level by means of various approaches. In some cases the Ritz method has been adopted due to its excellent tradeoff between accuracy-to-computational cost ratio. For instance Rao (1985) implemented a Ritz-based approach by adopting a Libove-Batdorf sandwich model in conjunction with plane stress constitutive law, while Kim and Hong (1988) considered the sandwich plate as the assembly of an anti-plane core, and two laminated faces connected by means of adhesive layers of finite bonding stiffness. The Ritz method is used also in the work of Hadi and Matthews (1998) in conjunction with a three-layers model in which facesheets and core are modeled according to the first-order shear deformation theory (FSDT). Fazzolari and Carrera (2013) studied the buckling of sandwich plates in the framework of a variable-kinematics approach. The Ritz approximation employed in the aforementioned works is built from trigonometric functions, which restricts the solution to simply-supported plates, and leads to strong inaccuracies in the presence of non-negligible amounts of bending/twisting coupling (Stone \& Chandler, 1996; Vescovini, Dozio, D'Ottavio, \& Polit, 2018; Wu, Raju, \& Weaver, 2012). Recently, Ritz-based approaches were developed for sandwich plates with variablestiffness faces based on FSDT (Coburn \& Weaver, 2016) and variable-kinematics models (Vescovini \& Dozio, 2016).

Other tools for global buckling analysis have been developed on the basis of a finite element approach. While the literature is relatively wide - a comprehensive overview can be found in the survey paper by Noor, Burton, and Bert (1996) -, significant examples are found in Babu and Kant $(1999,2000)$ where 2D elements are developed based on highorder equivalent single layer (ESL) model; third-order theory inclusive of zig-zag effects was implemented in $\mathrm{C}^{0}$ elements by Pandit, Singh, and Sheikh (2008).

Typical patterns of wrinkling-type instabilities render the solution of the problem particularly challenging. Indeed, refined spatial modeling is needed to properly capture the short half-wavelengths characterizing the buckling modes. For this reason, analytical strategies and design formulae have been the subject of several investigations. Well-known solutions for axially loaded isotropic panels were derived following the approaches outlined by Hoff (1945), Plantema (1966) and Allen (1969), see, e.g., the engineering design procedures proposed by Niu and Talreja (1999). Improved models accounting for face orthotropy can be found, among the relatively vast literature, in the analytical approaches due to Vonach and Rammerstorfer (2000), Birman and Bert (2004), Lopatin and Morozov (2008) and Koissin, Shipsha, and Skvortsov (2011). On the contrary, much less research has been devoted to the wrinkling analysis of sandwich panels with anisotropic faces. The analytical strategy proposed by Fagerberg (2003) and Fagerberg and Zenkert (2005a) is among the few to account for these effects, which can have a drastic impact on the panel response. In addition, it can be adopted for predicting the wrinkling response in the presence of multiaxial loading conditions.

Analytical strategies do generally provide a material strength interpretation of the wrinkling instabilities (Fagerberg \& Zenkert, 2005b), meaning that the phenomenon can be treated as a real material failure, independently from panel dimensions and boundary conditions. The reduced computational effort, which is essential during the early design steps, is another advantage provided by these strategies. At the same time, it should be noted that analytical approaches suffer a restricted range of applicability due to the many underlying assumptions that are necessary to render the problem tractable in a closed-form manner. In particular, they generally treat local buckling independently from the global one, thus excluding any possible interaction between these failure modes.

For these reasons, a useful and modern approach consists in developing design strategies capable of accurately predicting global and local buckling in a unified framework. Under the simplifying assumption of one-dimensional strut model, closedform solutions were derived by Léotoing, Drapier, and Vautrin (2002) based on CLT assumptions for facesheets and third 
order model for core. Exact solutions for global and local buckling of sandwich struts were derived referring to elasticity solutions (Ji \& Waas, 2007; 2008). D'Ottavio and Polit (2015) and D'Ottavio, Polit, Ji, and Waas (2016) obtained quasi-3D solutions for global buckling and wrinkling of uniaxially loaded orthotropic sandwich panels and struts by means of Naviertype solutions and high-order models formulated within a variable-kinematics approach. Two-dimensional structural models were considered by Rose, Moore, Knight, and Rankin (2002), where 2D sandwich elements were implemented in the finite element code STAGS and complemented with a strip-based feature for local buckling analysis. The Finite Strip Method was employed along with a three-layer model in Yuan and Dawe (2001a,b), where the faces are modeled as CLPT or FSDT plates and the core with a $\{1,2\}$ kinematics. Recently Khalili and Malekzadeh (2015) proposed a third order, fixed-kinematic approach in conjunction with a Navier solution procedure; a nonlinear strategy is proposed by Yu et al. (2015) based on finite element solution and a combined CLPT- $\{3,2\}$ kinematics for the faces and core, respectively.

Based on the previous literature survey, it appears that the availability of unified tools capable of dealing with local and global buckling instabilities of sandwich panels is still rather limited. The formulation presented in this paper relies upon the combined use of the Sublaminate Generalized Unified Formulation (SGUF) and the method of Ritz. It aims at providing a novel computational tool that can be successfully used for capturing both global and local instabilities, trying to conjugate the accuracy of detailed numerical computations with the efficiency of analytical solutions. The approach, due to D'Ottavio, Polit et al. (2016), extends the Generalized Unified Formulation (GUF) by introducing the possibility of defining sublaminates, each of them associated with a distinct polynomial representation, making it possible a drastic improvement of the accuracy-to-number of degrees of freedom ratio. SGUF is inscribed within the variable-kinematics framework of Carrera's Unified Formulation (CUF), whose formalism has been generalized in order to account for different kinematics models for different sublaminates, which is also the approach classically applied in sandwich theories, such as HSAPT/EHSAPT. However, contrarily to HSAPT/EHSAPT, the present approach does not rely upon kinematic assumptions which are defined ex ante. Any arbitrary kinematics can be defined, at sublaminate level, as an input of the analysis procedure. The possibility of deriving elegant, closed-form solution of HSAPT/EHSAPT models is thus lost, but the advantages are twofold. Firstly, improved refinement of core/faces can be achieved if needed - some test cases discussed in the paper will demonstrate this aspect. Secondly, the formulation is general enough to allow the analysis of those configurations which are not necessarily composed by the conventional sequence face-core-face. Examples can be found in the sandwich constructions studied by Cho and Averill (2000) or, recently, by Suzuki, Aoki, Ogasawara, and Fujita (2017). To a more general extent, the proposed tool can be easily employed for analysis purposes as new designs of sandwich structures become available, with no needs to modify the underlying theory.

The approach outlined in this paper is not intended as an alternative to finite element analyses, which, nowadays, can be conducted in a very effective way. The SGUF approach is aimed at providing a tool characterized by improved efficiency - the time for the analysis is of the order of few seconds -, allowing researchers to obtain upper-bound, highly-refined solutions that can be used for assessing design formulae or for benchmarking purposes. This latter aspect can be particularly useful in those cases where the derivation of elasticity solutions can be hard to achieve, such as in the case of anisotropic twodimensional plates. Furthermore, the availability of a variable-kinematic approach can be exploited to gather insight into the mechanical response of the panel, which could be more complex in the context of 3D finite element analyses.

The SGUF-Ritz approach is presented here in the context of a challenging case, given by the local/global buckling response of anisotropic sandwich panels, where finite element simulations would demand for highly refined mesh or local strip models. The formulation is effectively applied by considering multiaxial loading conditions, any kind of boundary conditions and accounting for the effects of face anisotropy.

\section{Modeling approach}

The sandwich plate considered in this paper occupies a volume $V=\Omega \times[-t / 2, t / 2]$, where $\Omega=[0, a] \times[0, b]$ is the mean surface lying on the Cartesian $\left(x_{1}, x_{2}\right)$-plane and $t$ is the uniform thickness along the direction $x_{3}=z$. For simplicity, but without loss of generality, a symmetric, single-core sandwich cross-section is considered that consists of an orthotropic core of thickness $t_{c}$. Each skin has a uniform thickness $t_{f}$ and consists of a stack of $N_{f}$ orthotropic plies. The principal material axes of each ply $k=1,2, \ldots, N_{s}$ are oriented at an angle $\theta_{k}$ with respect to the global $x_{1}$ direction. A sketch of the sandwich panel is provided in Fig. 1.

\subsection{Weak form of the linearized stability problem}

The weak form of the governing equations for the linearized stability analysis is obtained by application of the Trefftz criterion (Jones, 2006):

$$
\delta\left(\delta^{2} \Pi\right)=\int_{V}\left(\delta e_{i j} \tilde{c}_{i j l n} e_{l n}+\delta u_{l, \beta} \lambda \sigma_{\alpha \beta}^{0} u_{l, \alpha}\right) \mathrm{d} z \mathrm{~d} S=0,
$$

where $u_{i}$ and $e_{i j}$ are the components of the displacement vector and the compatible linear strain tensor, respectively, and $\delta$ indicates virtual variation. The buckling multiplier is denoted with $\lambda$, whilst $\sigma_{\alpha \beta}^{0}$ defines the prebuckling membrane state of stress. Note that, unless otherwise stated, Einstein's summation convention is adopted with Latin indices taken in $\{1,2,3\}$ and Greek indices in $\{1,2\}$. 


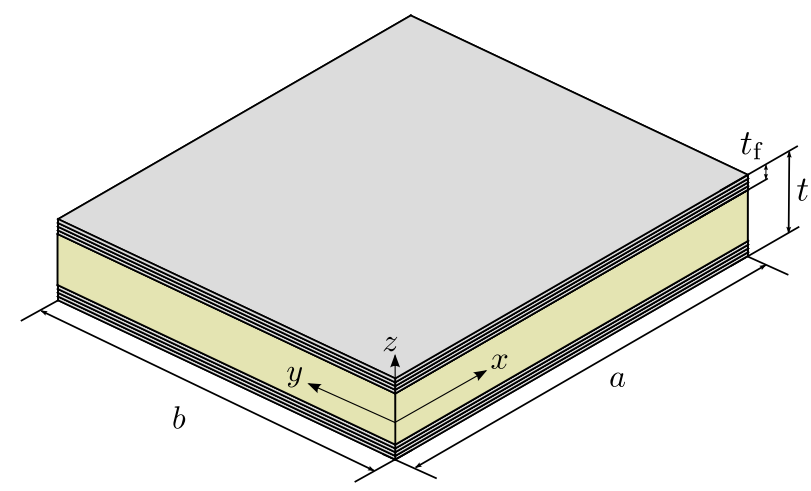

Fig. 1. Sandwich panel and reference system.

The constant stiffness moduli, expressed in the global Cartesian frame, are denoted as $\tilde{C}_{i j l n}$, while $\epsilon_{i j}$ is the workconjugate Green-Lagrange finite strain tensor defined as:

$$
\epsilon_{i j}=e_{i j}+\varepsilon_{i j} \text { with } e_{i j}=\frac{1}{2}\left(u_{i, j}+u_{j, i}\right) \text { and } \varepsilon_{i j}=\frac{1}{2} u_{l, i} u_{l, j} .
$$

The weak form in Eq. (1) can be expressed as function of the displacement components upon substitution of Eq. (2). The subsequent approximation of the displacement field allows provides the variationally consistent governing equations. On the one hand, approximations are associated with the chosen plate model, which postulates the dependence upon the thickness direction $z$ according to the following general expression

$$
u_{i}\left(x_{\alpha}, z\right)=\sum_{\tau=1}^{N_{u_{i}}} F_{\tau}(z) \hat{u}_{i_{\tau}}\left(x_{\alpha}\right) .
$$

The actual form of the assumed plate model is discussed in the next section. On the other hand, the Ritz approximation is introduced, which expresses the dependence on the in-plane coordinates $x_{\alpha}$ of the generalized displacement $\hat{u}_{i_{\tau}}$ :

$$
\hat{u}_{i_{\tau}}\left(x_{\alpha}\right)=\sum_{j=1}^{M} N_{u_{i} j}\left(x_{\alpha}\right) U_{i_{\tau j}} .
$$

Details about the chosen set of functions $N_{j}\left(x_{\alpha}\right)$ are reported in the section dedicated to the Ritz approximation. Once the approximation in Eqs. (3) and (4) are introduced into Eq. (1), all the derivatives and integrals can be explicitly evaluated and the following matrix form of the governing equations is eventually obtained:

$$
\delta \mathbf{U}^{\mathrm{T}}\left[\mathbf{K}+\lambda \mathbf{K}_{G}\right] \mathbf{U}=\mathbf{0}
$$

where $\mathbf{K}$ corresponds to the linear stiffness matrix and $\mathbf{K}_{G}$ to the geometric stiffness matrix related to the initial stress field. The solution of the linear eigenvalue problem expressed by Eq. (5) yields the critical load multipliers $\lambda$, the lowest of which defines the buckling load.

\subsection{Variable-kinematics plate models with SGUF}

The present variable kinematics modeling approach subdivides the composite stack composed of $N_{p}$ physical plies of thickness $t_{p}$, into $N_{k}$ computational sublaminates of thickness $t_{k}$. Each sublaminate is thus composed of $N_{p}^{k}$ plies and has a thickness $t_{k}=\sum_{p=1}^{N_{p}^{k}} t_{p}$, where the local ply index $p$ is introduced for each sublaminate. Hence, $t=\sum_{k=1}^{N_{k}} t_{k}=\sum_{k=1}^{N_{k}} \sum_{p=1}^{N_{p}^{k}} t_{p}$. Local ply-specific and sublaminate-specific thickness coordinates $z_{p} \in\left[-\frac{t_{p}}{2}, \frac{t_{p}}{2}\right]$ and $z_{k} \in\left[-\frac{t_{k}}{2}, \frac{t_{k}}{2}\right]$ are introduced along with the corresponding non-dimensional coordinates $\zeta_{p}=\frac{z_{p}}{t_{p} / 2}$ and $\zeta_{k}=\frac{z_{k}}{t_{k} / 2}$ for defining the approximations of the plate model. The link between these non-dimensional coordinates is expressed through following relation:

$$
\zeta_{p}=\frac{t_{k}}{t_{p}} \zeta_{k}+\frac{2}{t_{p}}\left(z_{0 k}-z_{0 p}\right)
$$

where $z_{0 p}$ and $z_{0 k}$ are the midplane coordinates of the $p$ th ply and $k$ th laminate, respectively.

The overall plate model is constructed as a Layer-Wise assembly of sublaminates. Each sublaminate is associated with a variable kinematics description that is defined according to the Generalized Unified Formulation proposed in Demasi (2009a, 2009b, 2009c): each displacement component $u_{i}$ of all plies of the $k$ th sublaminate can be expanded with arbitrary order $N_{u}^{k}$, and can be described in an ESL or LW manner. Due to this modeling strategy, different arbitrary kinematics can be independently chosen for the facesheets and the core of a sandwich plate. 
The displacement component $u_{i}^{p, k}\left(x_{\alpha}, z\right)$ of the $p$ th ply within the $k$ th sublaminate is expressed according to Eq. (3) as

$$
u_{i}^{p, k}\left(x_{1}, x_{2}, z_{p}\right)=\sum_{\alpha_{u_{i}}=0}^{N_{u_{i}}^{k}} F_{\alpha_{u_{i}}}\left(z_{p}\right) \hat{u}_{i \alpha_{u_{i}}}\left(x_{1}, x_{2}\right) \text {. }
$$

It is emphasized that the expansion order $N_{u_{i}}^{k}$ can be different depending on the component $i$ that is considered. For instance, in-plane displacements $u_{1}^{p, k}$ and $u_{2}^{p, k}$ may have a different expansion order than the out-of-plane displacement $u_{3}^{p, k}$. If the $k$ th sublaminate has a LW description, the resulting approximation over $z_{k}$ is obtained upon assembling the plyspecific approximations Eq. (7) across all $N_{p}^{k}$ plies through enforcing the continuity conditions at the interfaces between adjacent plies. If the sublaminate is described in an ESL sense, Eq. (7) still holds with the coordinate change $z_{p}=z_{k}$ and by setting $u_{i}^{p, k}=u_{i}^{k}$ and $\hat{u}_{i \alpha_{u_{i}}}^{p, k}=\hat{u}_{i \alpha_{u_{i}}}^{k}$.

The thickness functions $F_{\alpha_{u_{i}}}$ are defined in the non-dimensional coordinate $\zeta$, where $\zeta=\zeta_{p}$ if the sublaminate has an LW description and $\zeta=\zeta_{k}$ if the sublaminate has an ESL description. These functions are defined as follows

$$
\begin{array}{ll}
\text { if } N_{u_{r}}^{k}=0: & F_{0}(\zeta)=1 \\
\text { if } N_{u_{r}}^{k}>0: & F_{0}(\zeta)=\frac{1+\zeta}{2} ; \quad F_{1}(\zeta)=\frac{1-\zeta}{2} \\
& F_{l}(\zeta)=P_{l}(\zeta)-P_{l-2}(\zeta) \quad l=2,3, \ldots, N_{u_{r}}^{k}
\end{array}
$$

where $P_{l}(\zeta)$ is the Legendre's polynomial of order $l$ which is defined recursively as:

$$
P_{0}=1 ; \quad P_{1}=\zeta ; \quad P_{l+1}=\frac{(2 l+1) \zeta P_{l}-l P_{l-1}}{l+1}
$$

By virtue of these functions, the LW assembly procedures that enforces the displacement field continuity between adjacent plies and sublaminates can be accomplished in a straightforward manner (D'Ottavio, 2016; D'Ottavio, Dozio, Vescovini, \& Polit, 2016).

\subsection{Ritz method}

Within the Ritz solution framework, the functions $N_{j}\left(x_{\alpha}\right)$ that approximate the solution according to Eq. (4) should form a complete and admissible set of functions. In this work, two different sets of linearly independent approximating functions are proposed and compared. For the sake of generality, the physical domain $\Omega$ is conveniently mapped onto the computational domain $\Omega_{\square}=[-1,1] \times[-1,1]$, described by the natural coordinates $\xi$ and $\eta$. The approximating Ritz functions for the displacement component $u_{r}(r=1,2,3)$ are then expressed as

$$
N_{u_{r} j}(\xi, \eta)=\phi_{u_{r} m}(\xi) \psi_{u_{r} n}(\eta)
$$

$$
\text { with } m=1, \ldots, R ; \quad n=1, \ldots, S \text { and } j=S(m-1)+n
$$

where $R$ and $S$ are the orders of the expansion along $\xi$ and $\eta$, respectively, and $j=1, \ldots, M=R \times S$.

The first set of approximating functions consists of the product of a basis function and of a polynomial boundary function that allows to satisfy the essential conditions at the domain boundaries:

$$
\phi_{u_{r} m}(\xi)=f_{u_{r}}(\xi) p_{m}(\xi) ; \quad \psi_{u_{r} n}(\eta)=g_{u_{r}}(\eta) p_{n}(\eta)
$$

where the boundary functions are defined as:

$$
f_{u_{r}}(\xi)=(1+\xi)^{e_{1 r}}(1-\xi)^{e_{2 r}} ; \quad g_{u_{r}}(\eta)=(1+\eta)^{e_{1 r}}(1-\eta)^{e_{2 r}}
$$

and the coefficients $e_{1 r}$ and $e_{2 r}$ are set to either 0 or 1 depending on the boundary conditions (Dozio \& Carrera, 2012). The basis functions $p_{m}$ and $p_{n}$ are here chosen to be the orthogonal Legendre polynomials defined recursively by means of Eq. (9) with $l=m, n$ and $\zeta=\xi, \eta$. It is worth emphasizing that Legendre polynomials are characterized by excellent convergence properties and provide the highest degree of sparsity in the resulting stiffness matrix among the class of orthogonal polynomials (Vescovini et al., 2018).

For comparison purposes with the existing literature, a second set of approximating functions is considered, corresponding to the classical trigonometric Ritz expansion, taken from the well-known Navier solutions for plates simply-supported along the four edges. It consists of a complete basis that inherently verifies the essential boundary conditions of the 
problem, and can be cast in the form given by Eq. (10) as follows:

$$
\begin{aligned}
& N_{u_{1} j}(\xi, \eta)=\cos \frac{m \pi}{2}(\xi+1) \sin \frac{n \pi}{2}(\eta+1) \\
& N_{u_{2} j}(\xi, \eta)=\sin \frac{m \pi}{2}(\xi+1) \cos \frac{n \pi}{2}(\eta+1) \\
& N_{u_{3} j}(\xi, \eta)=\sin \frac{m \pi}{2}(\xi+1) \sin \frac{n \pi}{2}(\eta+1)
\end{aligned}
$$

It is important to note that the trigonometric basis functions do automatically impose some conditions on the derivatives of the solution at the boundaries. In particular, Eq. (14) implies vanishing curvatures at the supported edges, which meets well the natural boundary conditions of nil bending moments for isotropic and orthotropic plates. However, Eq. (14) imposes spurious over-constraints in the presence of bending-twisting coupling that characterizes the response of anisotropic plates, with detrimental effects over the convergence of the solution.

\subsection{Kernel integrals}

By virtue of the index notation employed in Eqs. (7) and (10) for expressing the displacement components along $z$ and in the plane $\Omega$, respectively, the variational statement Eq. (1) can be written in a compact manner in terms of so-called kernel integrals. It is worth noting that the kernel evaluation of the Ritz integrals allows for a highly efficient implementation, which is of paramount importance in wrinkling problems where several degrees of freedom need to be considered.

The integral across the thickness of the plate is represented as an assembly of integrals over sublaminates, which in turn consist of an assembly of integrals over the thickness of individual plies. A detailed derivation of these kernel integrals can be found in the literature dedicated to the variable kinematics approach (Vescovini et al., 2018), so in the following only the main equations are reported for the sake of conciseness. The approximations expressed by Eqs. (7) and (10) are introduced into Eq. (1) and, taking into account the definition of the compatible strains Eq. (2), the following discrete form of the variational statement is obtained

$$
\begin{aligned}
& \sum_{k=1}^{N_{k}} \sum_{p=1}^{N_{p}^{k}} \delta U_{r \alpha_{u_{r} i}}^{p, k} \tilde{C}_{R S}^{p, k} Z_{(\partial) u_{r}(\partial) u_{s}}^{p \alpha_{u_{s}} \beta_{u_{s}}} \mathcal{I}_{u_{r} u_{s} i j}^{\text {defg }} U_{s \beta u_{s} j}^{p, k}+\delta U_{r \alpha_{u_{r} i}}^{p, k} \lambda \sigma_{\gamma \mu}^{0 p, k} Z_{u_{r} u_{s}}^{p \alpha_{u_{r}} \beta_{u_{s}}} \mathcal{I}_{u_{r} u_{s} i j}^{\text {defg }} U_{s \beta u_{s} j}^{p, k}=0 \\
& \text { with } r, s=1,2,3 ; \gamma, \mu=1,2 ; \alpha_{u_{r}}=1,2, \ldots, N_{u_{r}}^{k} ; \beta_{u_{s}}=1,2, \ldots, N_{u_{s}}^{k} ; i, j=1,2, \ldots, M .
\end{aligned}
$$

$\tilde{C}_{R S}^{p, k}$ represents the generic $R S$ coefficient of the ply stiffness matrix expressed in the global reference system in Voigt notation

$$
\begin{aligned}
& \sigma_{R}^{p, k}=\tilde{C}_{R S}^{p, k} e_{S}^{p, k}(R, S=1,2, \ldots, 6) \\
& \text { with }\left\{\begin{array}{l}
e_{1}=e_{11}, e_{2}=e_{22}, e_{3}=e_{33}, e_{4}=2 e_{23}, e_{5}=2 e_{13}, e_{6}=2 e_{12} \\
\sigma_{1}=\sigma_{11}^{\prime}, \sigma_{2}=\sigma_{22}^{\prime}, \sigma_{3}=\sigma_{33}^{\prime}, \sigma_{4}=\sigma_{23}^{\prime}, \sigma_{5}=\sigma_{13}^{\prime}, \sigma_{6}=\sigma_{12}^{\prime}
\end{array}\right.
\end{aligned}
$$

where the notation $\sigma^{\prime}$ recalls that these are perturbation stresses. The terms $Z_{(\partial) u_{r}(\partial) u_{s}}^{p \alpha_{u_{r}} \beta_{u_{s}}}$ and $\mathcal{I}_{u_{r} u_{s} i j}^{\text {defg }}$ are the integrals of the thickness functions and of the Ritz functions, respectively, which are defined according to the following notation:

$$
\begin{aligned}
& Z_{u_{r} u_{s}}^{p \alpha_{u_{s}} \beta_{u_{s}}}=\int_{z_{p}^{\text {bot }}}^{z_{p}^{\text {top }}} F_{\alpha_{u_{r}}} F_{u_{u_{s}}} \mathrm{~d} z \quad Z_{\partial u_{r} u_{s}}^{p \alpha_{u_{r}} \beta_{u_{s}}}=\int_{z_{p}^{\text {bot }}}^{z_{p}^{\text {top }}} \frac{\partial F_{\alpha_{u_{r}}}}{\partial z} F_{\beta_{u_{s}}} \mathrm{~d} z \\
& Z_{u_{r} \partial u_{s}}^{p \alpha_{u_{r}} \beta_{u_{s}}}=\int_{z_{p}^{\text {bot }}}^{z_{p}^{\text {top }}} F_{\alpha_{u_{r}}} \frac{\partial F_{\beta_{u_{s}}}}{\partial z} \mathrm{~d} z \quad Z_{\partial u_{r} \partial u_{s}}^{p \alpha_{u_{r}} \beta_{u_{s}}}=\int_{z_{p}^{\text {bot }}}^{z_{p}^{\text {top }}} \frac{\partial F_{\alpha_{u_{r}}}}{\partial z} \frac{\partial F_{u_{u_{s}}}}{\partial z} \mathrm{~d} z \\
& \mathcal{I}_{u_{r} u_{s} i j}^{\text {defg }}=\int_{-1}^{1} \int_{-1}^{1} \frac{\partial^{d+e} N_{u_{r} i}}{\partial x^{d} \partial y^{e}} \frac{\partial^{f+g} N_{u_{s} j}}{\partial x^{f} \partial y^{g}} \mathrm{~d} \eta \mathrm{d} \xi \quad(d, e, f, g=0,1)
\end{aligned}
$$

\subsection{Prebuckling state}

The prebuckling stress condition is evaluated in a closed-form manner as first step of the analysis procedure. By assuming that the stress resultants $N_{\alpha \beta}^{0}$ are imposed at the sandwich edges, it is possible to determine the distribution of internal stress after imposing compatibility requirements between the various plies composing the plate. By assuming a prebuckling plane stress state, the membrane stiffness matrix of the plate $A_{I K}(I, K \in\{1,2,6\})$ and its inverse $a_{I K}=A_{I K}^{-1}$ are evaluated following the standard CLT procedure (Jones, 1998; Reddy, 2004). The prebuckling strains are then obtained as:

$$
\begin{aligned}
\epsilon_{x x}^{0} & =a_{11} N_{x x}^{0}+a_{12} N_{y y}^{0}+a_{16} N_{x y}^{0} \\
\epsilon_{y y}^{0} & =a_{12} N_{x x}^{0}+a_{22} N_{y y}^{0}+a_{26} N_{x y}^{0} \\
\gamma_{x y}^{0} & =a_{16} N_{x x}^{0}+a_{26} N_{y y}^{0}+a_{66} N_{x y}^{0}
\end{aligned}
$$


Table 1

Material elastic properties of the facesheets.

\begin{tabular}{llllll}
\hline & $F 1$ & $F 2$ & $F 3$ & $F 4$ & $F 5$ \\
\hline $\mathrm{E}_{11}(\mathrm{MPa})$ & 30,000 & 107,000 & 229,000 & 69,000 & 68,950 \\
$\mathrm{E}_{22}(\mathrm{MPa})$ & 30,000 & 15,000 & 13,350 & 69,000 & 68,950 \\
$\mathrm{E}_{33}(\mathrm{MPa})$ & 30,000 & 15,000 & 13,350 & 69,000 & 68,950 \\
$\mathrm{G}_{12}(\mathrm{MPa})$ & 11,538 & 4300 & 5249 & 26,538 & 26,519 \\
$\mathrm{G}_{13}(\mathrm{MPa})$ & 11,538 & 4300 & 5249 & 26,538 & 26,519 \\
$\mathrm{G}_{23}(\mathrm{MPa})$ & 11,538 & 4300 & 3000 & 26,538 & 26,519 \\
$v_{12}$ & 0.30 & 0.30 & 0.3151 & 0.30 & 0.30 \\
$v_{13}$ & 0.30 & 0.30 & 0.3151 & 0.30 & 0.30 \\
$v_{23}$ & 0.30 & 0.30 & 0.3151 & 0.30 & 0.30 \\
\hline
\end{tabular}

Table 2

Material elastic properties of the cores (F: foam; H-C: honeycomb).

\begin{tabular}{llllllllllll}
\hline & $\mathrm{C} 1$ & $\mathrm{C} 2$ & $\mathrm{C} 3$ & $\mathrm{C} 4$ & $\mathrm{C} 5$ & $\mathrm{C} 6$ & $\mathrm{C} 7$ & $\mathrm{C} 8$ & $\mathrm{C}$ & $\begin{array}{l}\mathrm{C} 10 \\
\mathrm{H}\end{array}$ \\
Type & $\mathrm{F}$ & $\mathrm{F}$ & $\mathrm{H}-\mathrm{C}$ & $\mathrm{F}$ & $\mathrm{F}$ & $\mathrm{F}$ & $\mathrm{F}$ & $\mathrm{F}$ & $\mathrm{H}-\mathrm{C}$ & $\mathrm{H}-\mathrm{C}$ & $\mathrm{H}-\mathrm{C}$ \\
\hline $\mathrm{E}_{11}(\mathrm{MPa})$ & variable & variable & $\approx 0$ & 40.00 & 80.00 & 140.00 & 20.00 & 56.00 & $\approx 0$ & 0.6895 & $\approx 0$ \\
$\mathrm{E}_{22}(\mathrm{MPa})$ & - & - & $\approx 0$ & 40.00 & 80.00 & 140.00 & 20.00 & 56.00 & $\approx 0$ & 0.6895 & $\approx 0$ \\
$\mathrm{E}_{33}(\mathrm{MPa})$ & 46.15 & 86.00 & $+\infty$ & 40.00 & 80.00 & 140.00 & 20.00 & 56.00 & 109.00 & 68.95 & 828.0 \\
$\mathrm{G}_{12}(\mathrm{MPa})$ & - & - & $\approx 0$ & 16.00 & 32.00 & 56.00 & 13.00 & 22.00 & $\approx 0$ & 0.265 & $\approx 0$ \\
$\mathrm{G}_{13}(\mathrm{MPa})$ & 11.54 & 21.50 & 146 & 16.00 & 32.00 & 56.00 & 13.00 & 22.00 & 26.60 & 82.74 & 146.0 \\
$\mathrm{G}_{23}(\mathrm{MPa})$ & - & - & 90.40 & 16.00 & 32.00 & 56.00 & 13.00 & 22.00 & 15.50 & 49.64 & 90.4 \\
$v_{12}$ & - & - & 0.00 & 0.25 & 0.25 & 0.25 & 0.25 & 0.25 & 0.00 & 0.3 & 0.00 \\
$v_{13}$ & 0.25 & 0.25 & 0.00 & 0.25 & 0.25 & 0.25 & 0.25 & 0.25 & 0.00 & 0.01 & 0.00 \\
$v_{23}$ & - & - & 0.00 & 0.25 & 0.25 & 0.25 & 0.25 & 0.25 & 0.00 & 0.01 & 0.00 \\
\hline
\end{tabular}

The corresponding prebuckling stresses are determined in each ply from the constitutive law

$$
\begin{aligned}
& \sigma_{x x}^{0, p, k}=C_{11}^{p, k} \epsilon_{x x}^{0}+C_{12}^{p, k} \epsilon_{y y}^{0}+C_{16}^{p, k} \gamma_{x y}^{0} \\
& \sigma_{y y}^{0, p, k}=C_{12}^{p, k} \epsilon_{x x}^{0}+C_{22}^{p, k} \epsilon_{y y}^{0}+C_{26}^{p, k} \gamma_{x y}^{0} \\
& \sigma_{x y}^{0, p, k}=C_{16}^{p, k} \epsilon_{x x}^{0}+C_{26}^{p, k} \epsilon_{y y}^{0}+C_{66}^{p, k} \gamma_{x y}^{0}
\end{aligned}
$$

where $C_{I K}^{p, k}$ denote the reduced plane stress stiffness coefficients of the $p$ th ply.

Two different strategies are considered here for evaluating the prebuckling stresses. The first one consists in applying Eqs. (19) and (20) as reported, thus accounting for the contribution of the facesheets and core to the overall axial stiffness of the laminate. This approach simulates the case of plate loaded by means of rigid blocks, where the load is introduced both in the face plies and the core, according to their relative stiffnesses.

The second approach considers a load introduction mechanism where the core remains unloaded. In this case the application of Eqs. (19) and (20) is formally identical, but the core contribution is removed from the evaluation of the compliance matrix $a_{I K}$ and the index $p$ of Eq. (20) varies over the plies belonging to the facesheets only. This second load introduction strategy resembles the well-known anti-plane stress assumptions used for deriving wrinkling closed-form solutions.

\section{Results}

A number of test cases is illustrated to show the potentialities of the proposed formulation as a unified mean for addressing global and local instabilities of sandwich plates. Comparisons are illustrated against reference results from the literature as well as closed-form design formulae. Global buckling is addressed first, while local instabilities are illustrated in the second part of the discussion.

As far as several examples are presented, the material elastic properties used throughout this section are preliminarily summarized in Tables 1 and 2. The materials are labeled as Fi and Ci to denote the facesheet and core properties, respectively.

The notation for specifying the underlying kinematic theory is based on two acronyms, the first referring to the model adopted for the facesheet, the second relative to the core. Since all the examples considered here involve symmetric sandwich plates, the top and bottom facesheets are always modeled using the same theory. Furthermore, all sublaminate models employed in the subsequent examples are characterized by expansion orders $N_{u_{1}}^{k}=N_{u_{2}}^{k}=N_{u_{3}}^{k}+1$, i.e., they form a consistent approximation of order $N_{u_{3}}^{k}$ of the transverse shear strain $\gamma_{\alpha 3}^{k}$. These choices are, however, not mandatory within the present implementation. For instance, $\mathrm{ESL}_{110} / \mathrm{ESL}_{332}$ defines a model where the facesheet sublaminate is described by means of an Equivalent Single Layer theory of order 110 - i.e., an FSDT kinematics with plane stress constitutive law and a unit shear correction factor -, while the core is modeled according to a $\{3,2\}$ kinematics, in which the in-plane displacements 


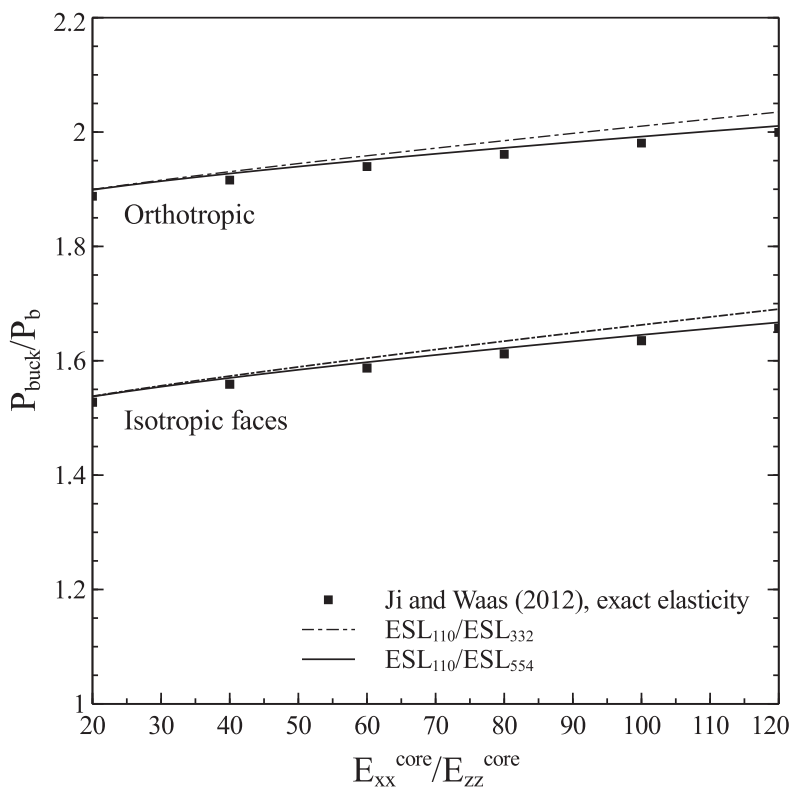

Fig. 2. Global buckling load of a sandwich strut - comparison against exact elasticity solution.

are cubic and the transverse displacement quadratic along the thickness. The full 3D constitutive law is used whenever the polynomial order used for the transverse displacement is greater than one.

\subsection{Global buckling}

The first two examples are aimed at assessing the accuracy of the predictions obtained with SGUF-Ritz approach in case of global buckling modes, i.e. modes characterized by halfwaves of length similar to the in-plane dimensions of the structure.

\subsubsection{Example 1 - thick sandwich strut with orthotropic core}

The test case is taken from Ji and Waas (2012), who derived the exact elasticity solution for a sandwich strut subjected to simply support conditions at the two ends, and loaded with an imposed end shortening. The strut aspect ratio $a / t$ is equal to 3 , where $t$ denotes the overall thickness of the strut, and $a$ is the total length. The thickness of the facesheets is equal to $0.1 \mathrm{~mm}$, while the core is $0.8 \mathrm{~mm}$ thick. Two different configurations are analyzed: in the first case the facesheets are made of the isotropic material F1 and the core of the orthotropic material C1; the second case refers to orthotropic facesheets made of material F2 and the orthotropic core C2. Note that in both configurations the core stiffness along the $x$ direction is larger in comparison to the stiffness along the transverse direction $z$.

The strut model is inherently two-dimensional and defines a plane problem, associated here with the $x z$ plane. The corresponding Ritz model is realized as a degeneration of the general framework previously outlined by dropping out the contributions due to the derivatives along the $y$ direction. The model is thus reduced to a cylindrical bending problem, and the Ritz description is based upon the approximation of the generalized displacement components along the strut axis only. The results are summarized in Fig. 2, where the non-dimensional buckling load is plotted against the orthotropy ratio of the core material. The reference value $P_{b}$ denotes the buckling load calculated as:

$$
P_{b}=\frac{P_{e}}{1+P_{e} / G A}
$$

where $P_{e}$ is the Euler buckling load, and GA the strut transverse shear stiffness according to Bazant and Beghini (2006).

All the configurations buckle with a global shape, characterized by one single halfwave along the strut axis. The results are presented by considering two different models, namely $E S L_{110} / E S L_{332}$ and $E S L_{110} / E S L_{554}$, and a number of Ritz functions equal to 10 is used following a preliminary numerical assessment. The comparison against the exact results of Ji and Waas (2012) reveals the convergence with respect to the theory order. As seen, using the high-order model 332 for the core can be inadequate for accurately predicting the buckling loads, especially for relatively high values of core orthotropy ratios, i.e., when the in-plane stiffness of the core is much larger than that in the thickness direction. Close matching is observed with the reference results when the core model is refined up to the order 554, as seen from Fig. 2. Further refinement of the kinematic theory, both for the facesheets and core, does not provide any additional benefit, as the reduction of the buckling load is below $0.3 \%$. In this sense the model $E S L_{110} / E S L_{554}$ represents the optimal ratio between accuracy and number of degrees of freedom. As expected, the results obtained with the present Ritz approach lead to buckling loads which are slightly higher in comparison to the exact elasticity solution. 


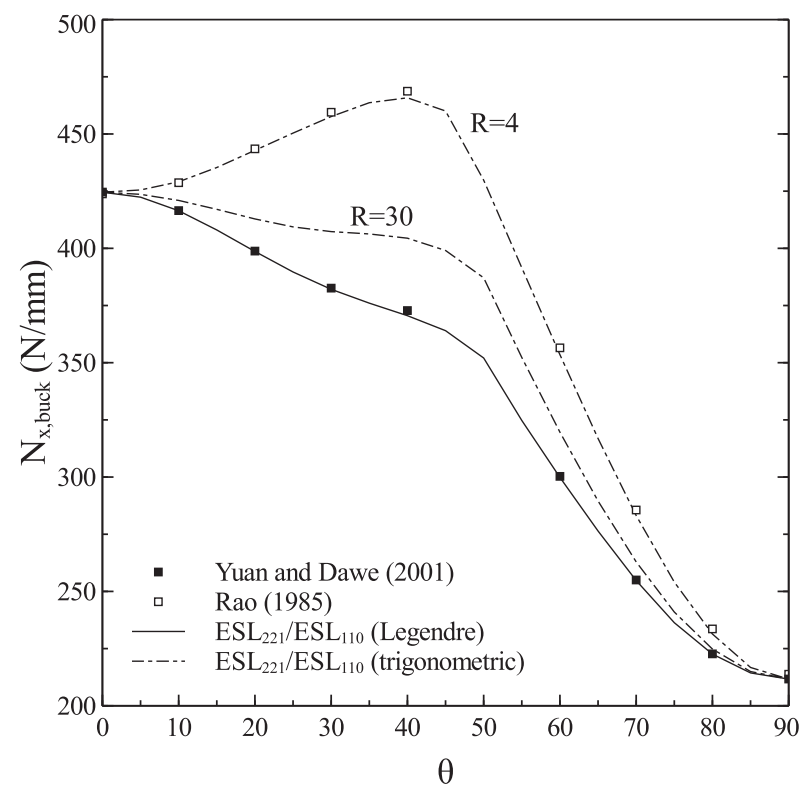

Fig. 3. Global buckling load of axially loaded sandwich plate - effect of different shape functions.

This preliminary example provides good evidence of the advantages due to the sublaminate variable-kinematics approach, where the core model can be refined up to the desired level of accuracy depending on the problem at hand.

\subsubsection{Example 2 - sandwich panel with honeycomb core}

The second example deals with the test case proposed by Rao (1985) and Yuan and Dawe (2001b). Simply-supported square panels of dimension $225 \mathrm{~mm}$ are considered. Uniaxial loading conditions are introduced by imposing a uniform compressive strain at the facesheets, while assuming identically null the prebuckling stress in the core. The facesheets are $0.2 \mathrm{~mm}$ thick and made of one single ply (material F3) oriented at $\theta$, while the honeycomb core (material C3) has thickness equal to $10 \mathrm{~mm}$.

The present configuration is characterized by membrane and bending anisotropy when $\theta$ is different from $0^{\circ}$ and $90^{\circ}$. This feature renders the Ritz solution of particular interest, as Navier-type results cannot be achieved unless anisotropy is assumed negligible. Based on a preliminary convergence analysis, 30 trial functions are used along both the coordinate directions $x$ and $y$. Consistently with the B-spline finite strip formulation of Yuan and Dawe (2001a), the model $\mathrm{ESL}_{110} / \mathrm{ESL}_{221}$ is adopted for the present computations. The results are reported in Fig. 3, where the buckling force per unit length is plotted against the orientation $\theta$ of the facesheet. A first set of results is obtained by expanding the displacement field by means of Legendre polynomials that, due to their excellent convergence properties, is the default approach considered here. The predicted buckling loads are reported with a continuous line and are in close agreement with the reference results, with a maximum percent difference below $0.6 \%$. It is worth noting that no appreciable improvement is achieved by further refining the underlying kinematic theory. With the aim of illustrating the effects of an improper choice of the trial functions, the results are calculated also by considering a trigonometric expansion in the form of Navier-type functions. The buckling loads are plotted with dash-dotted lines in Fig. 3, by considering 4 functions, as done by Rao (1985), and 30 functions. In the first case, close agreement is noticed with the buckling loads reported by Rao (1985) that are, however, much higher with respect to the correct predictions. Even by increasing the number of trial functions up to 30 , the errors are still relatively large, especially for those configurations characterized by high degree of bending/twisting elastic coupling. The fallacy of trigonometric functions is related to their inability to fulfill the natural boundary conditions in the presence of bending anisotropy as well as their incompleteness in the strain energy norm (Stone \& Chandler, 1996; Vescovini et al., 2018).

\subsection{Wrinkling under uniaxial loads}

This second section is directed toward the analysis of wrinkling-type instabilities, thus the SGUF-Ritz approach is adopted for assessing the response of sandwich panels undergoing buckling modes characterized by wavelengths of the order of the panel thickness. It should be noticed that honeycomb cores are modeled as homogeneous orthotropic materials, such that intracellular buckling ("dimpling") of the facesheets is excluded from the present approach.

\subsubsection{Example 3 - sandwich strut with foam core}

Aiming at further highlighting the capabilities of the formulation, an initial benchmark from the literature is illustrated. The problem was previously investigated by Ji and Waas (2007) that derived the exact elasticity solution. For this reason, 


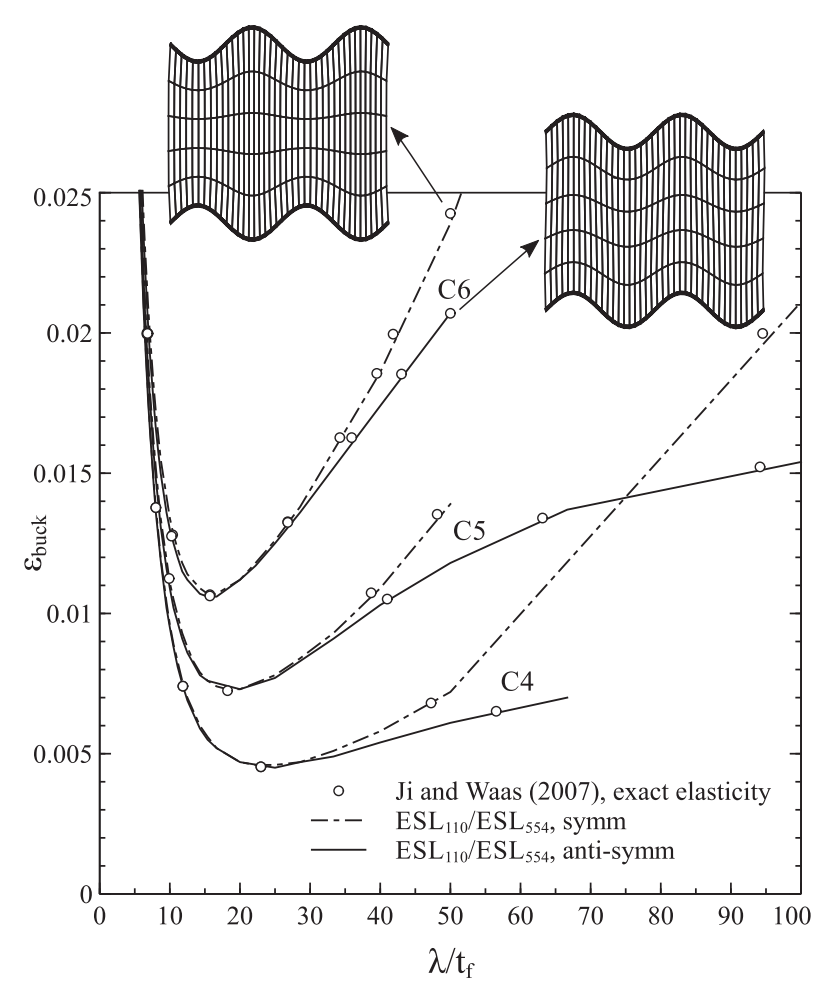

Fig. 4. Symmetrical and antisymmetrical local buckling loads of a sandwich strut for different core properties - comparison against exact elasticity solution.

the comparison is believed of special importance. The sandwich construction is a simply-supported strut of length equal to $200 \mathrm{~mm}$; the thicknesses of each facesheet and the core are equal to $1 \mathrm{~mm}$ and $68 \mathrm{~mm}$, respectively. Three distinct core properties are considered, corresponding to foam cores with different densities and denoted here as C4, C5 and C6. The elastic constants of the facesheet are derived by evaluating the equivalent modulus and Poisson's ratio of the crossply stack $[0 / 90]_{s}$ of material F2 that are found to be equal to $61.44 \mathrm{GPa}$ and 0.0738 , respectively (D'Ottavio, Polit et al., 2016). Uniaxial loading conditions are imposed by means of an imposed strain along the whole strut section. The analysis is conducted to identify both symmetric and antisymmetric wrinkling modes associated with different wavelengths. In this case, convergence of the results is obtained by considering a model $\mathrm{ESL}_{110} / \mathrm{ESL}_{554}$.

The plot of Fig. 4 reports the axial strain at buckling against the non-dimensional half-wavelength of the wrinkling mode $\lambda / t_{f}$, where $\lambda$ is the half-wavelength and $t_{f}$ the thickness of the facesheet. Within the present Ritz implementation, the wrinkling half-wavelength cannot be imposed ex ante, as the displacement field is the superposition of several trial functions. For this reason, the problem is solved for a relatively large expansion - up to 150 function -, and 150 eigenvalues are computed. For each eigenvalue, the corresponding eigenvector is post-processed in order to establish the half-wavelength and to check whether it is of symmetric or antisymmetric type. The plot reported in Fig. 4 is the collection of the obtained points. It is worth noting that the eigenvalues tend to be clustered in the short wavelength regime, thus the overall reference curve cannot be exactly reproduced. However, the results demonstrate the close agreement between the approximate SGUF solution and the exact elasticity one. Also the distinction between symmetric and antisymmetric modes is properly described, which confirms that the method correctly captures the through-the-thickness behavior. For the sake of illustration, Fig. 4 also shows the symmetric and antisymmetric buckling modes predicted at $\lambda / t_{f}=50$ for the configuration with the $\mathrm{C6}$ core.

\subsubsection{Example 4 - sandwich panel with foam core}

Having illustrated the capability of the method to obtain quasi-3D predictions for the wrinkling response, the analysis is now shifted to a more complex case characterized by the presence of facesheets with anisotropic behavior. With this regard, the amount of data available in the literature is relatively poor, and few numerical and experimental results are available for comparison purposes. The sandwich panel studied analytically and experimentally by Fagerberg (2003) is considered: it has planar dimensions $200 \mathrm{~mm}$ and $150 \mathrm{~mm}$ along the longitudinal and transverse directions, respectively. The facesheets consist of a symmetric laminate of four plies (material F2, thickness $0.25 \mathrm{~mm}$ ) with stacking sequence $[\alpha /(90+\alpha)]_{s}$. The angle $\alpha$ defines the rotation of the orthotropy principal axes and varies between $0^{\circ}$ and $90^{\circ}$. Therefore, the faces are specially orthotropic if $\alpha$ equal to $0^{\circ}$ or $90^{\circ}$, while different amounts of flexural anisotropy are associated with the remaining configurations, depending on the magnitude of the off-axis angle $\alpha$. The core is $50 \mathrm{~mm}$ thick and is made of material C7. 


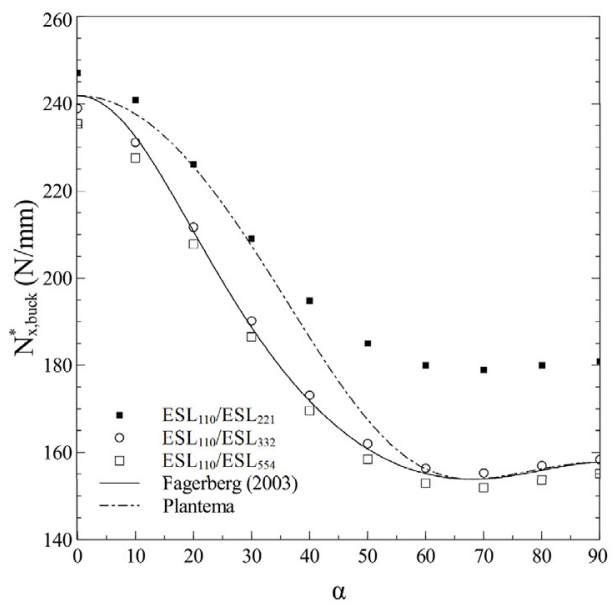

(a)

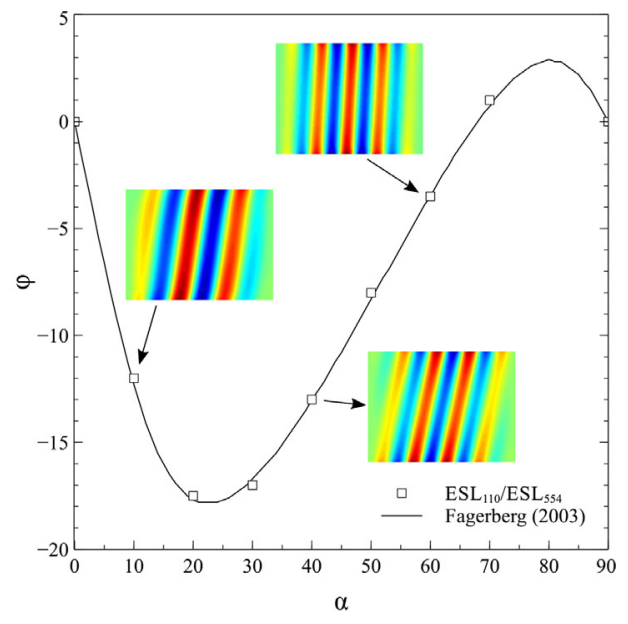

(b)

Fig. 5. Local buckling response of axially loaded foam-core sandwich plate with anisotropic faces and comparison against closed-form solutions: (a) buckling loads and (b) skew angle of the buckled pattern.

In order to reproduce the effect of the tabs used for the load introduction, the panel is assumed fully clamped along the transverse edges, and free on the longitudinal ones; the load is introduced by means of an imposed axial strain. The results are summarized in Fig. 5, where the critical load and the skew angle of the wrinkles are reported for different values of $\alpha$. Note that, for consistency with the results reported in Fagerberg (2003), the load $N_{x \text { buck }}^{*}$ is defined as half of the buckling force per unit length carried by the panel. The same notation is adopted hereinafter. The buckling loads obtained using the SGUF-Ritz approach are reported with respect to different kinematic theories: FSDT models are assumed for the facesheet, whilst the core model is progressively refined by increasing the order of the theory. From Fig. 5(a) it can be noted that highly unconservative buckling loads are predicted if the lowest order kinematic theory, corresponding to that proposed by Yuan and Dawe (2001a), is used for the core. Even the third order model 332 is responsible for buckling loads 2\% higher with respect to the converged results, which are achieved using a 554 core model. Again, the advantages due to the adoption of a variable-kinematics approach, whose levels of refinement can be defined form case to case, are quite evident.

Fig. 5(a) reports also the results obtained by the closed-form solution proposed by Fagerberg and Zenkert (2005a) and the classical one due to Plantema (1966). Both these models rely upon an assumed exponential decay of the wrinkling mode along the thickness direction. While Plantema's model is based on the assumption of wrinkling modes perpendicular to the load direction, the solution due to Fagerberg and Zenkert accounts for the skewness of the wrinkling mode. Close matching between the present Ritz solution and the closed-form solution by Fagerberg and Zenkert can be noticed by inspection of Fig. 5(a). For any value of $\alpha$, the analytical approach leads to slightly higher buckling loads with respect to those obtained with the converged Ritz solution. On the contrary, Plantema's solution reveals a certain inaccuracy, always on the unsafe side, for those lay-ups characterized by an important amount of flexural anisotropy with angles $\alpha$ comprised in the range between $10^{\circ}$ and $60^{\circ}$.

Finally, it is interesting to have a closer look at the shape of the wrinkling modes by comparing the skewness angle as predicted by the model of Fagerberg and Zenkert and the Ritz solution. The results are reported in Fig. 5(b), where the buckling pattern is presented for a selected set of panels. A substantial agreement is noted, both in terms of trend of the curve as well as numerical values.

\subsection{Wrinkling under biaxial loads}

Wrinkling modes due to biaxial loading conditions have been quite rarely addressed in past efforts in the literature, especially for sandwich panels with anisotropic facesheets. For this reason, a deep analysis is presented here, both with respect to the assessment of the underlying kinematic theory, and in terms of comparison against available closed-form results.

The sandwich configurations considered in this section are taken from Fagerberg and Zenkert (2005a), among the few authors to address, both analytically and experimentally, the role of biaxial loads and facesheet anisotropy. The sandwich panels have dimension $200 \mathrm{~mm} \times 150 \mathrm{~mm}$; the core (material C8) has a thickness of $50 \mathrm{~mm}$, while the facesheet is made of one orthotropic ply of material F2 and thickness $0.5 \mathrm{~mm}$. Different orientation angles $\theta$ of the facesheet ply are considered. The boundary conditions are modeled by assuming the panel as fully clamped, while biaxial loads are introduced by means of imposed strains at the four edges. Note that the load is introduced both in the facesheets and the core. 
Table 3

Buckling loads $(\mathrm{N} / \mathrm{mm})$ for biaxially compressed panels: effects of face ply orientation and biaxiality ratio. Face theory $E S L_{110}$, and various core theories; $50 \times 50$ functions.

\begin{tabular}{|c|c|c|c|c|c|c|c|c|c|}
\hline \multirow[b]{2}{*}{ Core theory } & \multicolumn{3}{|l|}{$r=0.0$} & \multicolumn{3}{|l|}{$r=0.5$} & \multicolumn{3}{|l|}{$r=1.0$} \\
\hline & $\mathrm{ESL}_{332}$ & $\mathrm{ESL}_{554}$ & ESL $_{776}$ & $\mathrm{ESL}_{332}$ & $\mathrm{ESL}_{554}$ & $\mathrm{ESL}_{776}$ & $\mathrm{ESL}_{332}$ & ESL $_{554}$ & $\mathrm{ESL}_{776}$ \\
\hline \multicolumn{10}{|l|}{$\theta$} \\
\hline 0 & 227.31 & 214.19 & 213.38 & 229.64 & 214.87 & 213.05 & 168.57 & 134.08 & 131.14 \\
\hline 15 & 222.13 & 205.03 & 203.97 & 214.94 & 185.78 & 183.42 & 168.47 & 133.99 & 131.05 \\
\hline 30 & 207.73 & 181.33 & 179.51 & 196.32 & 163.62 & 161.00 & 168.38 & 133.90 & 130.96 \\
\hline 45 & 190.50 & 158.09 & 155.58 & 182.23 & 148.28 & 145.49 & 168.39 & 133.89 & 130.96 \\
\hline 60 & 177.59 & 143.06 & 140.23 & 173.54 & 139.08 & 136.21 & 168.40 & 133.90 & 130.97 \\
\hline 75 & 171.31 & 136.32 & 133.39 & 169.93 & 135.18 & 132.27 & 168.45 & 133.96 & 131.02 \\
\hline 90 & 169.97 & 135.08 & 132.15 & 169.59 & 134.83 & 131.91 & 168.51 & 134.03 & 131.12 \\
\hline
\end{tabular}

\subsubsection{Theory assessment}

An assessment over the kinematic model is performed for illustrating the roles of biaxiality ratio and facesheet anisotropy in relation to the order of the theory to guarantee accurate predictions. The results are summarized in Table 3, where the local buckling loads are reported by considering different facesheet orientations $\theta$ and values of the ratio $r=N_{y} / N_{x}$. The comparison is presented for different core models, while keeping fixed the kinematics used for the facesheet. In particular, a preliminary assessment, not reported here for the sake of conciseness, revealed that a facesheet model ESL 110 is always adequate. Furthermore, the core model $\mathrm{ESL}_{776}$ was found to guarantee convergence of the predictions. Therefore, the results obtained using the model $\mathrm{ESL}_{110} / \mathrm{ESL}_{776}$ are taken as reference.

As seen from Table 3, large errors are obtained when the core is modeled using 332 kinematics, with percent differences ranging between $7 \%$ and $29 \%$ with respect to the reference results. These results, obtained with a kinematic model similar to that of EHSAPT, provide good evidence of the advantages offered by the proposed approach when facing some class of problems. Note that the magnitude of errors is not negligible even when the core is modeled using a 554 kinematics, with values as high as $2 \%$. In both cases, the errors increase by moving along the rows and the columns of the table. In other words, the need for increased core refinement is determined by larger values of $r$ and $\theta$. This behavior is explained by noticing that higher amounts of biaxiality ratio tend to reduce the half-wavelengths of the wrinkles. Similarly, the facesheet stiffness along the axial direction is progressively weakened as the orthotropic plies are rotated, and the half-wavelengths of the corresponding mode are reduced. The onset of shorter wrinkles, in turn, determines a more rapid decay of the wrinkling perturbation through the core thickness. So, as the biaxiality ratio $r$ and rotation angle $\theta$ are increased, the core deformation is confined into a decay region that becomes thinner, which calls for a refinement of the core model kinematics. This behavior is demonstrated by the plots of Fig. 6: the curves report the normalized transverse and axial displacements of the core at the buckle crest obtained for different biaxiality ratios and ply angles. The plots are obtained using the high-order kinematics 776 to model the core. For $\theta=0^{\circ}$, an increasing decay rate can be observed in the transverse displacement curves of Fig. 6(a) if the ratio $r$ is increased from 0 to 1 . The axial displacement shows, in turn, an increasingly non-linear behavior, with the steepest gradient occurring next to the facesheets within a region that becomes narrower as the biaxiality ratio increases. For $\theta=45^{\circ}$, the curves in each plot are close to each other, and all of them are characterized by relatively steep gradients in the top and bottom regions adjacent to the facesheets, even in the case of pure axial load ( $r=0)$, see Fig. 6 (b). This also explains the large percent errors observed in Table 3 when $\theta=45^{\circ}$, irrespective on the loading condition.

\subsubsection{Further remarks on the refinement of the theory}

Further insight into the role played by the kinematic model employed for the core can be gained through the analysis of the strain energy contributions of the wrinkled configuration. The non-dimensional strain energy contributions can be evaluated, for each sublaminate $k$, as

$$
\begin{aligned}
\bar{U}_{\alpha \beta}^{k} & =\frac{1}{2 U_{t o t}} \int_{V^{k}} \sigma_{\alpha \beta} \epsilon_{\alpha \beta} \mathrm{d} V^{k} \\
\bar{U}_{33}^{k} & =\frac{1}{2 U_{t o t}} \int_{V^{k}} \sigma_{33} \epsilon_{33} \mathrm{~d} V^{k} \\
\bar{U}_{\alpha 3}^{k} & =\frac{1}{2 U_{t o t}} \int_{V^{k}} \sigma_{\alpha 3} \gamma_{\alpha 3} \mathrm{~d} V^{k} \quad \text { with } \alpha, \beta=1,2
\end{aligned}
$$

where no summation is implied now for the repeated indexes.

The results are shown in Fig. 7 for the biaxial case $(r=0.5)$ and facesheet ply orientation $\theta=45^{\circ}$ of Table 3 . The internal energy distribution is compared with respect to the different core models previously considered. Again, the lowest order kinematic is taken as $\mathrm{ESL}_{110} / \mathrm{ESL}_{332}$ to resemble the assumptions of classical high-order sandwich panel models, and providing evidence of the need for improved refinement for the test case at hand. As observed, most of the energy is stored in the core in the form of normal and transverse shear deformation energies. The presence of both these contributions highlights a wrinkling mode which is not purely extensional along the thickness. It follows that refinement is needed for 

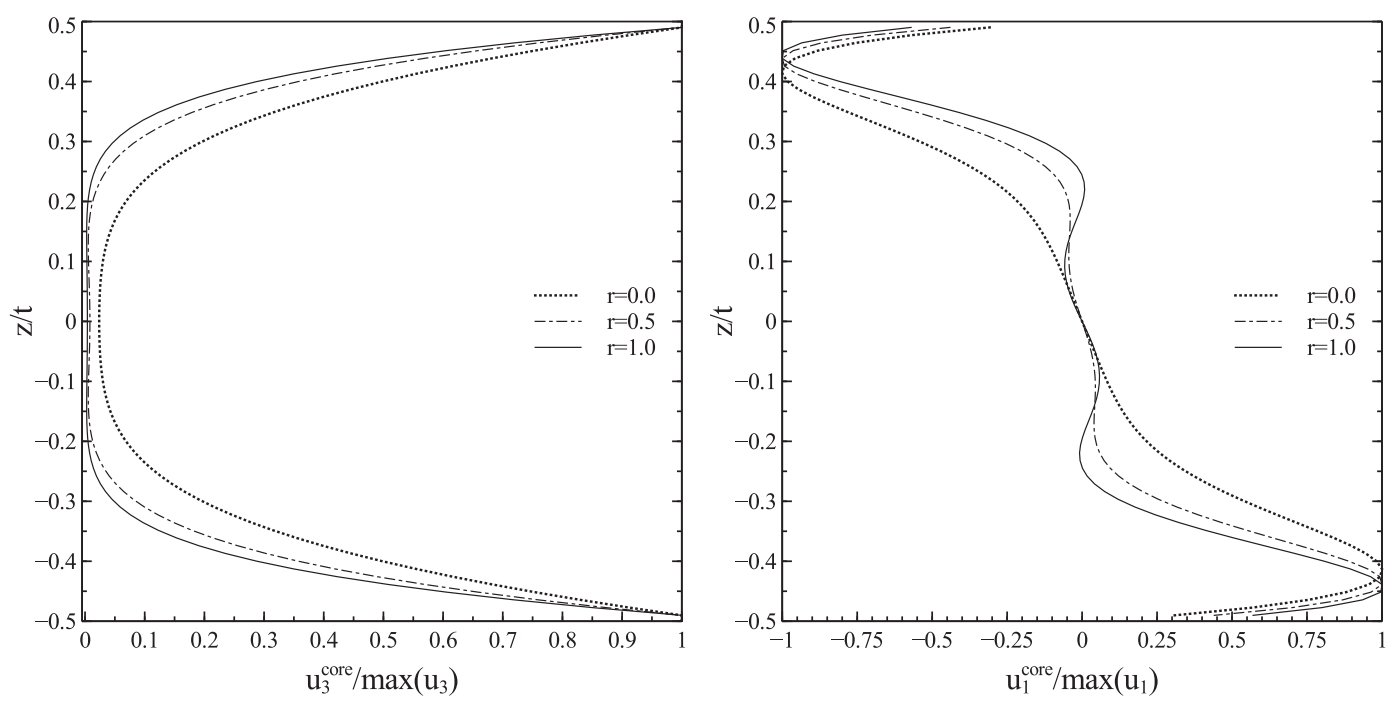

(a) face ply angle $\theta=0^{\circ}$
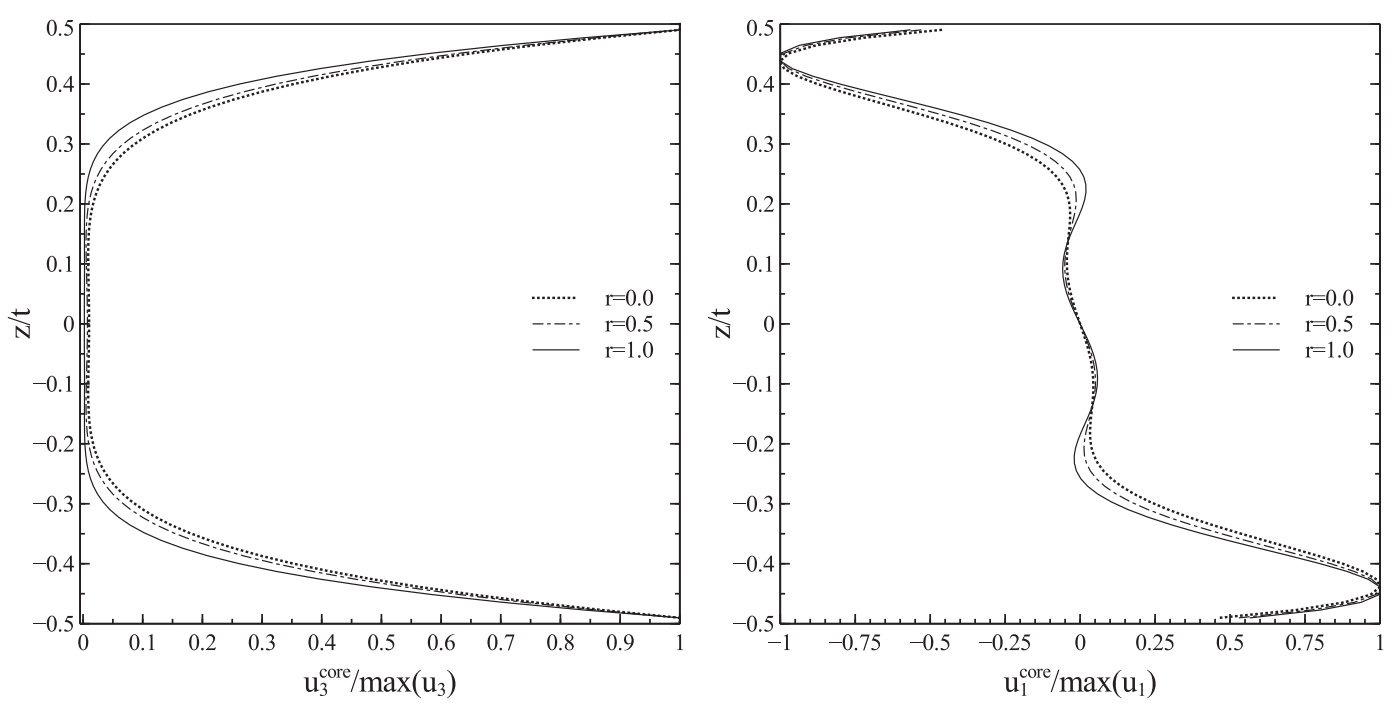

(b) face ply angle $\theta=45^{\circ}$

Fig. 6. Through-the-thickness transverse and axial displacements at the buckle crest for different biaxiality ratios $r$.

both, the displacement component $\mathrm{u}_{3}$, which is strictly associated with the transverse normal deformation energy $\bar{U}_{33}$, and the components $u_{1}$ and $u_{2}$, which are related to the transverse shear deformation energy $\bar{U}_{\alpha 3}$. The comparison between different sandwich models reveals that low-order core models tend to alter the internal load path by overestimating the transverse shear deformation mechanisms. This is due to the incapability of the models to properly describe the vertical displacement along the thickness, and so the correct amount of extensional energy $\bar{U}_{33}$ cannot be stored. This, in turn, determines a spillover of strain energy from $\bar{U}_{33}$ to the contributions $\bar{U}_{\alpha 3}$, creating an internal load path dominated by transverse shear mechanisms.

These considerations clearly explain the need for a high-order theory for correctly predicting the wrinkling behavior of sandwich panels with isotropic cores. These remarks are next extended to a broader class of configurations, in particular by including the core orthotropy ratio $E_{11}^{\text {core }} / E_{33}^{\text {core }}$. Preliminary investigations indicated a high sensitivity of the internal strain energy distribution to this parameter (D'Ottavio \& Polit, 2015). Present results refer to the same sandwich panel investigated above, but the in-plane elastic moduli of the core C 8 are now varied, while keeping constant $E_{33}^{\text {core }}$. The results are plotted in Fig. 8(a) in terms of non-dimensional transverse normal and transverse shear energy contributions for core's orthotropy ratios between 1 (isotropic) and $10^{-5}$. The configuration with $r=1$ and $\theta=45^{\circ}$ has been computed with the high-order 


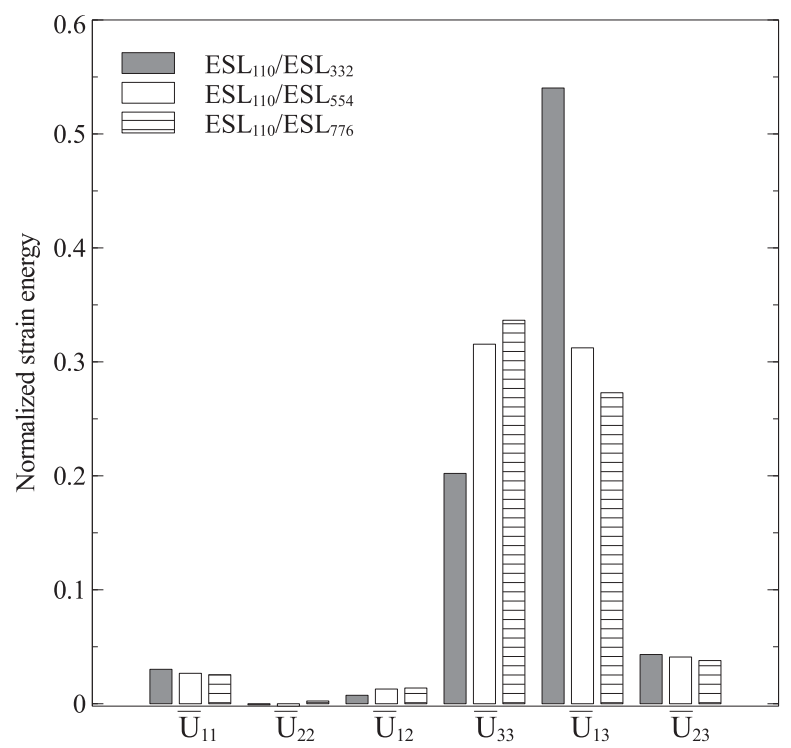

Fig. 7. Normalized core strain energies for biaxial load $(r=0.5)$ and face ply angle $\theta=45^{\circ}$.

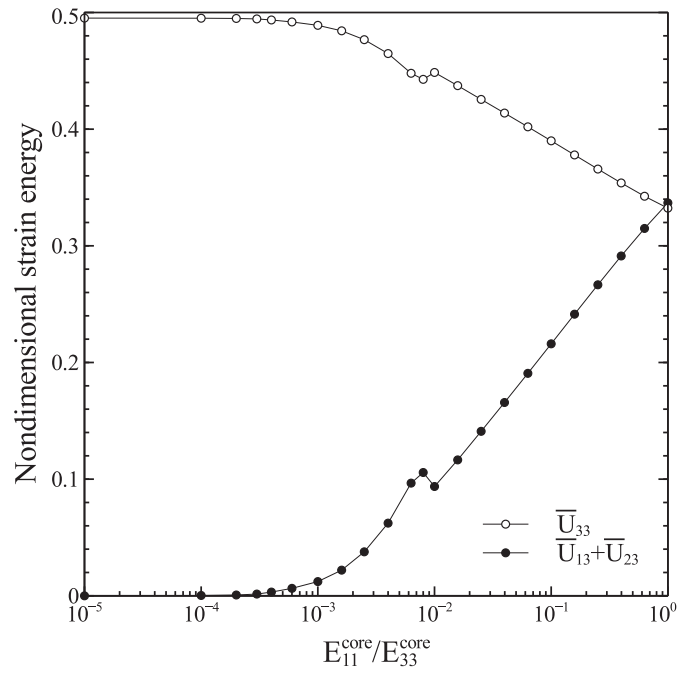

(a)

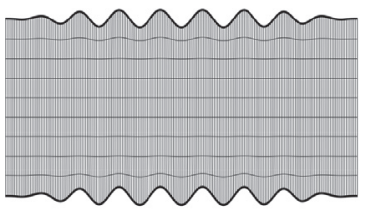

(b) $E_{11}^{\text {core }} / E_{33}^{\text {core }}=1$

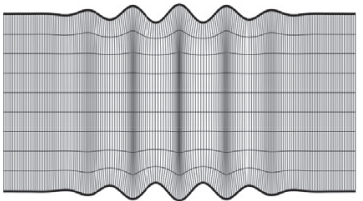

(d) $E_{11}^{\text {core }} / E_{33}^{\text {core }}=6 \cdot 10^{-3}$

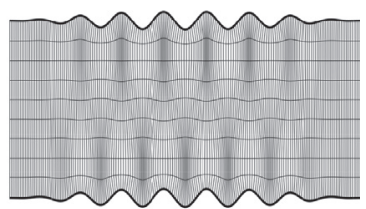

(c) $E_{11}^{\text {core }} / E_{33}^{\text {core }}=10^{-2}$

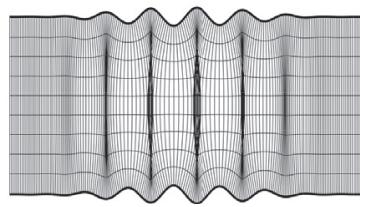

(e) $E_{11}^{\text {core }} / E_{33}^{\text {core }}=10^{-4}$

Fig. 8. Effect of core anisotropy for biaxial load $\left(r=1\right.$ ) and face ply angle $\theta=45^{\circ}$ : (a) normalized strain energies in the core, (b)-(e) wrinkling modes at $y=b / 2$.

model $\mathrm{ESL}_{110} / \mathrm{ESL}_{776}$. Fig. 8 illustrates also the deformed cross-sections of the wrinkling modes associated with four values of the core's orthotropy ratio.

The results show that weak in-plane properties tend to determine purely extensional core mechanisms. Hence, for approximately $E_{11}^{\text {core }} / E_{33}^{\text {core }}<10^{-3}$, the kinematic model is only required to correctly grasp the transverse normal strain in order to guarantee accurate wrinkling predictions. In this case, it turns out that the classical Winkler model provides fairly accurate results (Vonach \& Rammerstorfer, 2000) and, accordingly, low order models yield accurate results as well. For instance, the core model $\mathrm{ESL}_{221}$, which entails transverse normal strains and transverse shear strains that are constant and linear, respectively, across the core thickness, is typically sufficient to provide accurate results. On the contrary, wrinkling of isotropic cores, like that investigated in Table 3, is characterized by an equal repartition of the transverse normal and shear strain energies. As previously discussed, this configuration demands a core model based on high-order kinematics for capturing the interaction between the transverse normal and shear deformations within the decay region of the core.

The wrinkled cross-section illustrated in Fig. 8(b) shows that no interaction occurs between the facesheets for an isotropic core. In fact, the decay region is small compared to the core thickness $t_{c}$ and the buckling loads for antisymmetric and 


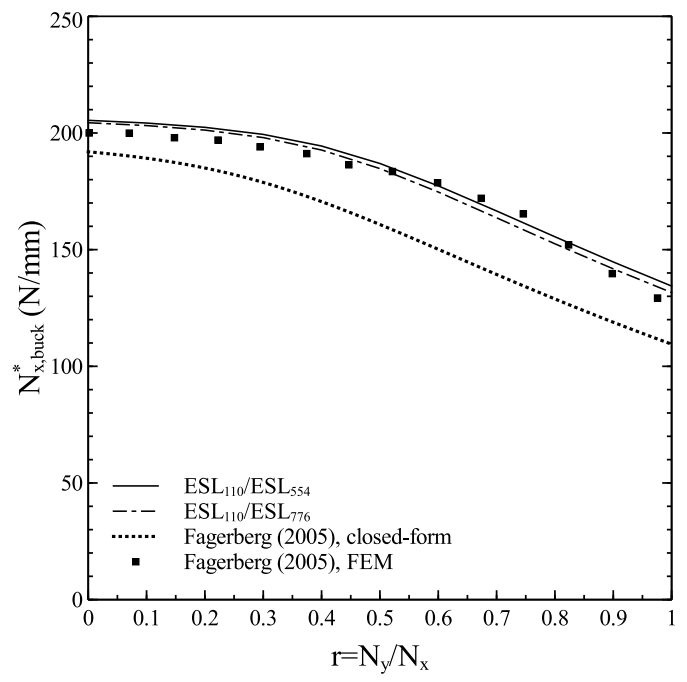

(a)

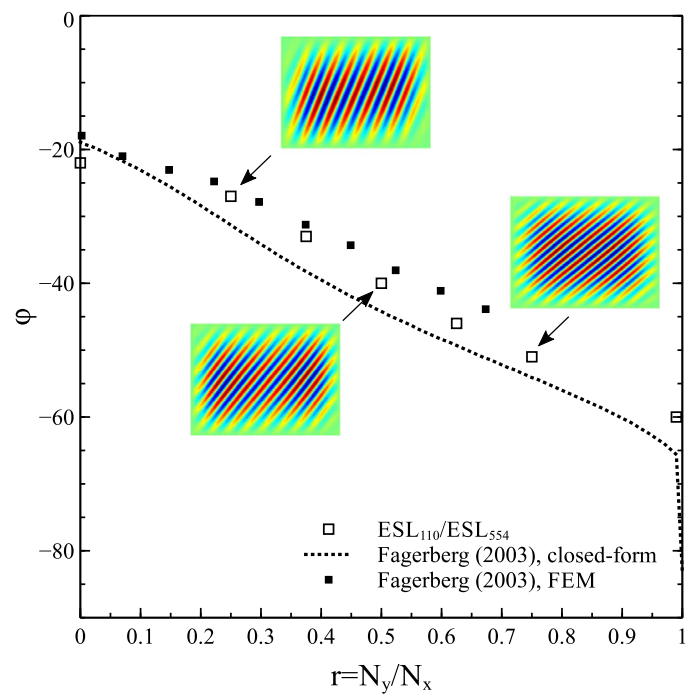

(b)

Fig. 9. Local buckling response of biaxially loaded soft-core sandwich plate with ply face oriented at $\theta=15^{\circ}-$ comparison against $3 \mathrm{D}$ FEM and closed-form solutions: (a) buckling loads, (b) skew angle of the buckled pattern.

symmetric short wavelength wrinkling modes are practically coincident for isotropic cores (see, e.g., Niu \& Talreja, 1999; Vonach \& Rammerstorfer, 2000). Starting from the isotropic case, the decrease of the core's in-plane moduli extends the decay region and, hence, stimulates the interaction between the facesheets. For the considered core material, the interaction is mainly driven by the transverse shear strains, which thus triggers a predominant antisymmetric mode as illustrated in Fig. 8(c). A further reduction of the core's in-plane stiffness enhances the interaction between the facesheets, which is now triggered mainly by the axial strains. The predominant wrinkling mode becomes the symmetric one, as seen from Fig. 8(d) and (e). The transition between symmetric and antisymmetric wrinkling modes can also be observed in Fig. 8(a) because the variation of the strain energy contributions with respect to the core's orthotropy ratio is linear for the antisymmetric mode and nonlinear for the symmetric mode.

\subsubsection{Interaction curves}

The stability region for combined biaxial loads can be quickly traced by making use of the present SGUF-Ritz implementation. This feature is particularly useful for realizing design charts in the form of interaction curves. With this regard, the sandwich panel of the previous example is analyzed for different values of the biaxiality ratio $r$ and ply angle equal to $15^{\circ}$. The results are summarized in Fig. 9, where the comparison is presented against the Fagerberg closed-form solution, both in terms of buckling loads and skew angle of the buckling half-waves. The curves report also the results of detailed 3D finite element analyses taken from Fagerberg and Zenkert (2005a). Good agreement can be noticed between the finite element predictions and the present SGUF-Ritz solutions, the latter providing, in general, slightly higher buckling loads, exception made for the range $0.6<r<0.8$. These discrepancies are attributed to the different definition of the boundary conditions utilized in FEM and Ritz analyses, the former allowing the in-plane shear, the latter preventing it. On the contrary, despite the comparison with the analytical solution reveals a similar trend, both for the buckling loads and the predicted skew angle, significant differences are obtained in terms of predicted values. The percent differences for the buckling loads range between $6 \%$ and $11 \%$. The discrepancies are attributed to the assumptions of the closed-form solution over the periodicity of the buckled pattern, which is not observed in the Ritz (see Fig. 9(b)) and FEM results.

Additional results are presented in Fig. 10 for different orientations of the facesheet ply. Also in this case the SGUF-Ritz results are very close to detailed 3D FEM analyses, while the closed-form solution describes a similar trend, but smaller buckling loads. It is interesting to highlight the close matching between Ritz and closed-form results in the initial region of the specially orthotropic panel of Fig. 10(a). This configuration is characterized by a buckling pattern with by zero skew angle and short-wave wrinkles along both directions, which verifies fairly well the periodicity assumption the closed-form solution relies upon. On the contrary, in presence of anisotropy a buckling patterns arises with short waves along one single direction, thus leading to a buckling phenomenon at panel level that is inherently different from the periodic one assumed by the closed-form solution. 


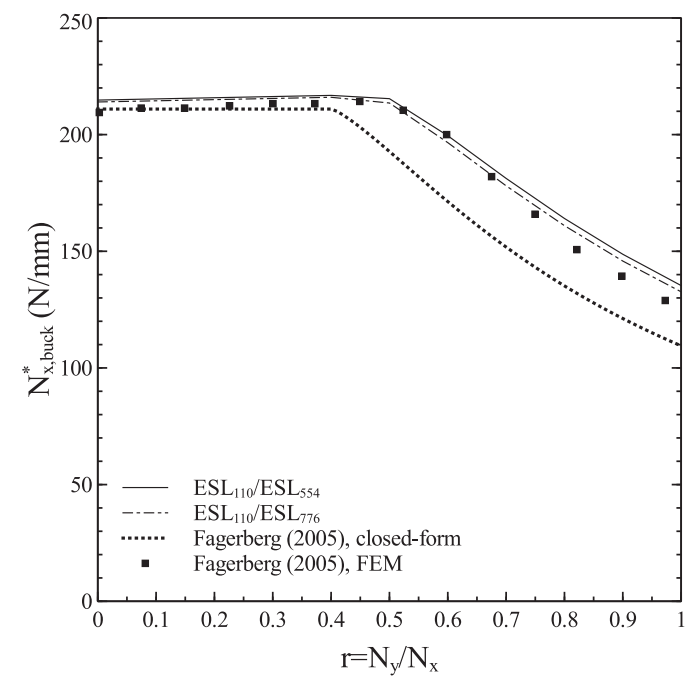

(a)

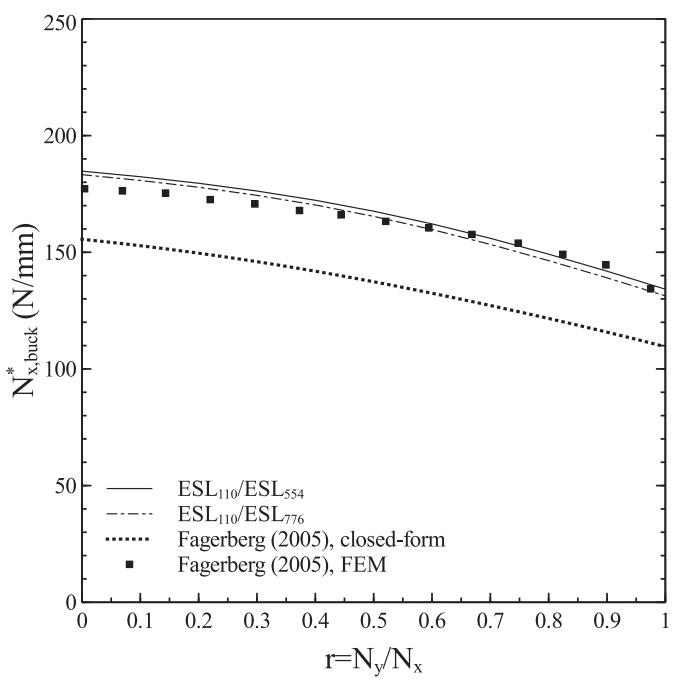

(b)

Fig. 10. Local buckling response of biaxially loaded soft-core sandwich plate for different ply face orientations and comparison against 3D FEM and closedform solutions: (a) $\theta=0^{\circ}$ and (b) $\theta=30^{\circ}$.

\subsection{Wrinkling under multi-axial loads}

In this section the results are presented for general loading conditions, where in-plane shear loads are introduced in addition to biaxial compression.

\subsubsection{Example 5 - isotropic sandwich panel with honeycomb core}

A first example is aimed at demonstrating the capability of the present implementation to handle in-plane shear loads. The example is taken from Yuan and Dawe (2001b) and deals with a simply-supported square sandwich plate of edge length $228 \mathrm{~mm}$, whose facesheets (material F4) and honeycomb core (material c9) are 0.65 and 25 mm thick, respectively. The prebuckling condition consists of longitudinal compression and in-plane shear and is introduced by loading the plate facesheets.

The shear-compression interaction curve is plotted in Fig. 11(a), and Ritz results are reported against detailed finite strip calculations reported by Yuan and Dawe (2001b). Ritz calculations are performed by means of $50 \times 50$ functions, using the model $\mathrm{ESL}_{110} / \mathrm{ESL}_{221}$, which corresponds to that adopted in the reference. The comparison reveals almost identical results, thus demonstrating the possibility of successfully applying the present approach to the analysis of more complex loading conditions. It is worth noting that no appreciable difference is observed if the order of the theory is refined. Since the honeycomb core has a null in-plane stiffness, the wrinkling mode is purely extensional along the thickness direction and no transverse shear energy is stored in the core. The kinematics order 221 adopted for the core is thus sufficient to properly capture the buckling mechanism. A sketch of wrinkling modes is available in Fig. 11(a), where the top view is shown for different ratios between compression and shear, and in Fig. 11(b)-(d), where the through-the-thickness behavior is reported along the $x$-coordinate at $y=b / 2$.

\subsubsection{Example 6 - composite sandwich panel with foam core}

Novel results are reported by considering the sandwich panel studied by Fagerberg and Zenkert (2005a) and analyzing the wrinkling behavior for combined loads of shear and biaxial compression. The dimensions of the panel are $200 \times$ $150 \mathrm{~mm}^{2}$, each facesheet consists of one $0.5 \mathrm{~mm}$ thick ply (material F2) oriented at $\theta$ and the core (material C8) has a thickness equal to $50 \mathrm{~mm}$. The panel is fully clamped at the four edges and the prebuckling condition is achieved by loading the sandwich panel by means of imposed strains, uniformly distributed across the sandwich thickness.

This test case is more challenging than the previous one, as the buckling mechanism involves both extensional and transverse shear energies. It follows that the core description demands for a high-order theory. A sandwich model ESL ${ }_{110} / \mathrm{ESL}_{776}$ was found adequate to guarantee convergence and is thus used for performing the calculations.

The results are summarized in Fig. 12, where the buckling forces per unit length are presented in the classical form of interaction curves. Two configurations are considered, namely an orthotropic panel with the ply constituting the facesheets oriented at $\theta=0^{\circ}$, and a strongly anisotropic panel whose facesheets are oriented at $45^{\circ}$. The interaction curves reported in Fig. 12(a) are traced by considering axial compression and shear, those reported in Fig. 12(b) refer to a prebuckling condition consisting of biaxial compression and shear. The results provided by the Fagerberg closed-form solution are included 


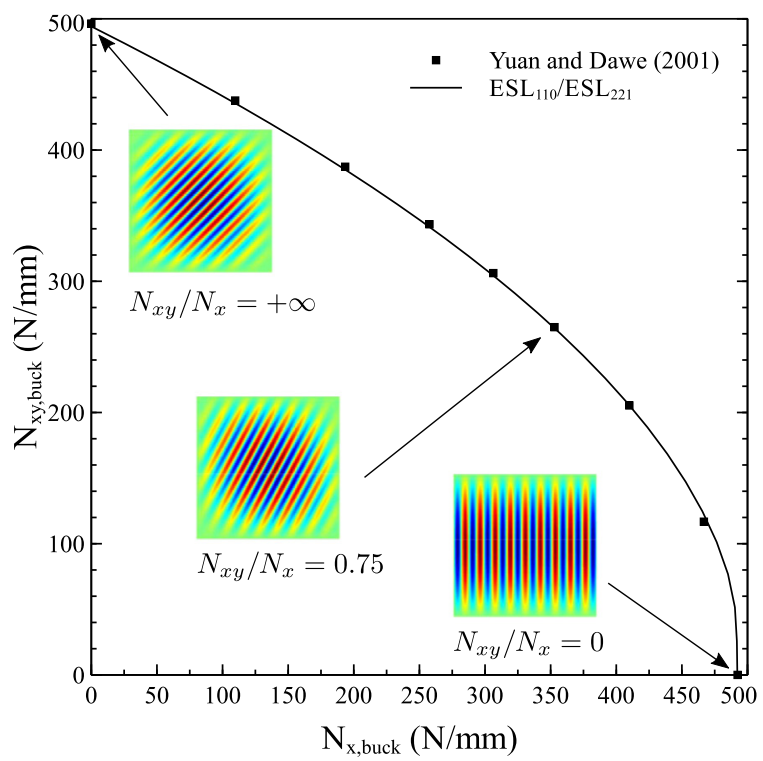

(a)

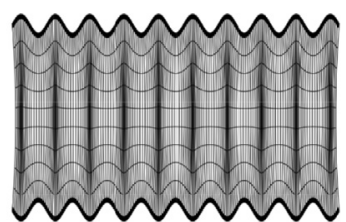

(b) $N_{x y} / N_{x}=0$

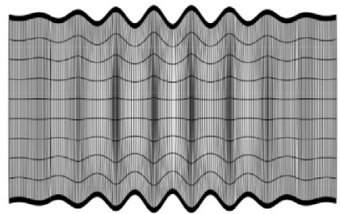

(c) $N_{x y} / N_{x}=0.75$

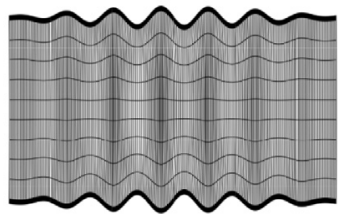

(d) $N_{x y} / N_{x}=+\infty$

Fig. 11. Local buckling behavior of isotropic sandwich plate with honeycomb core, loaded with combined shear and compression: (a) interaction curve and (b)-(d) wrinkling modes at $y=b / 2$.

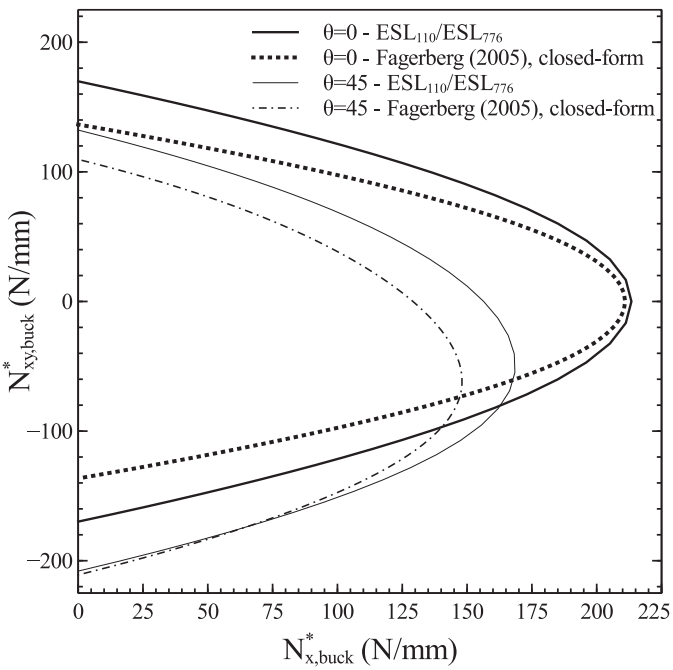

(a)

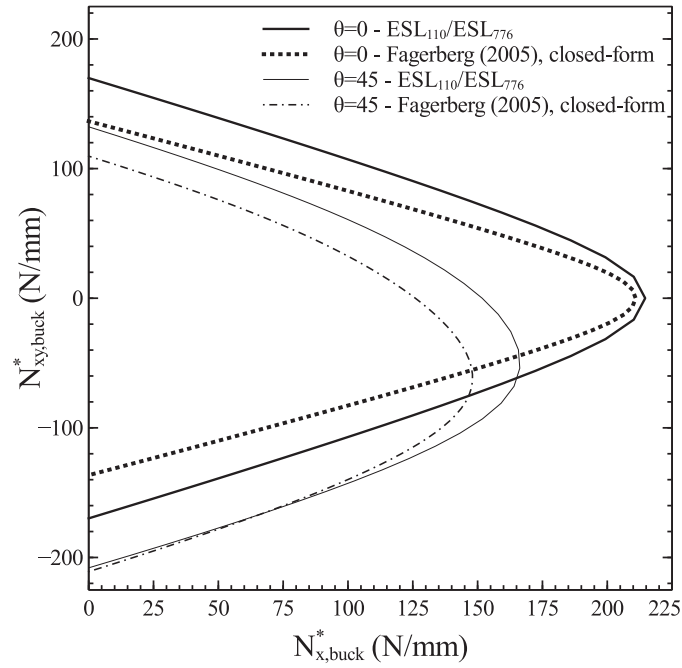

(b)

Fig. 12. Local buckling behavior of composite, foam-core sandwich plate, loaded with combined shear and compression and: (a) $N_{y}=0$ and (b) $N_{y}=0.25 N_{x}$.

for comparison purposes. For the orthotropic configuration, the interaction curves are symmetric with respect to the $x$ axis because the sign of the shear load has no influence on the buckling response. It is worth noting the good agreement between the present results and the closed-form solution for purely axial conditions, while larger discrepancies appear for increasing amounts of shear. In the case of the anisotropic configuration, the sign of the shear load has a strong influence on the buckling response and, as a result, the interaction curves are not symmetric with respect to the horizontal axis. The differences between the present Ritz solution and the analytical closed-form solution are small for negative values of shear, corresponding to compressive loads along the fiber direction. On the contrary, positive shear determines compressive loads 


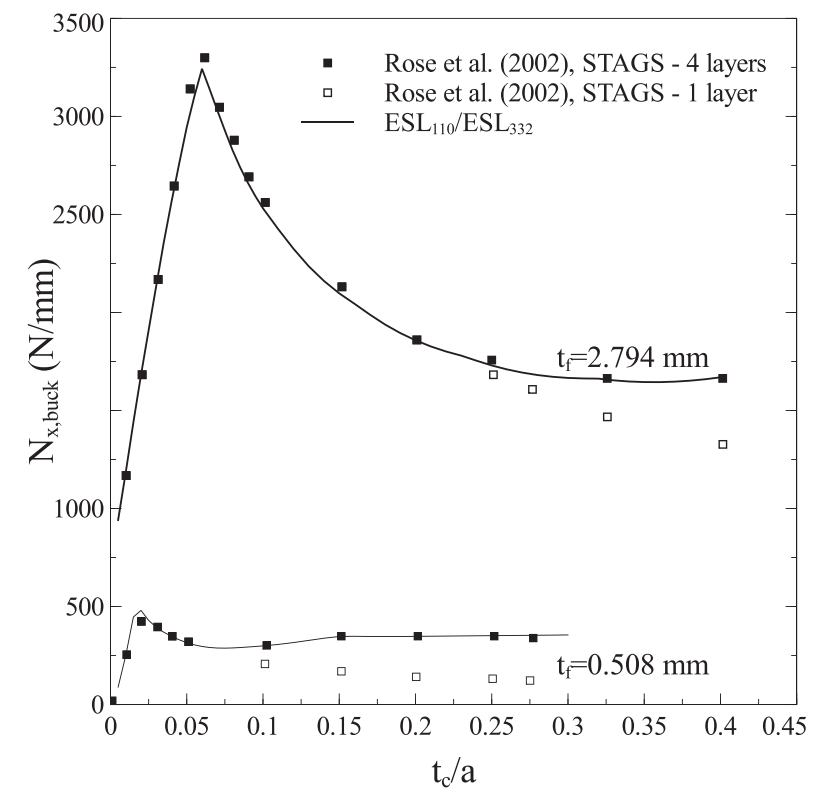

Fig. 13. Transition from global to local buckling response: isotropic sandwich plate with honeycomb core.

along the weaker in-plane direction, which yields larger discrepancies according to the findings highlighted in the biaxial load case.

\subsection{Transition from global buckling to wrinkling}

The main advantage of a unified approach for the buckling analysis, as the one outlined here, relies upon the possibility of capturing both global and local instabilities within the context of the same framework. This feature becomes especially useful when performing sensitivity studies or parametric analyses. Indeed, the modification of one or more design parameters of a sandwich construction may lead to a change of the buckling mechanism, which may turn from global to local. In some cases, the coupled use of distinct analysis models was suggested for analyzing global buckling and local buckling separately. In the context of finite element simulations, this need is mainly dictated by the computational burden associated with the large number of degree of freedom associated with highly refined meshes. This need is prevented by the adoption of the present Ritz approach, where both global and local buckling modes can be effectively captured with a reduced computational effort, and convergence is achieved quickly thanks to the excellent properties of the Legendre polynomials.

Two examples illustrating the transition from global to local instabilities are reported in Figs. 13 and 14. Note that the results extracted from the original sources have been put in a consistent form, reporting the force per unit length against the non-dimensional parameter $t_{c} / a$.

The results reported in Fig. 13 refer to a case study proposed by Rose et al. (2002), which involves a rectangular sandwich panel of dimensions $508 \times 254 \mathrm{~mm}^{2}$, whose facesheets are made of aluminum (material F5), while the core is the honeycomb denoted as C10. The panel is constrained with simply-supported conditions along the four edges, and loaded with uniaxial compression. For consistency with the finite element models discussed by Rose et al. (2002), a uniform strain is applied at the facesheets, thus leading to a prebuckling state where no stresses are carried by the core. Fig. 13 reports the buckling loads for two different values of the facesheet thickness, i.e., a thin face with $h_{f}=0.508 \mathrm{~mm}$ and a thick face with $h_{f}=2.794 \mathrm{~mm}$. The present SGUF-Ritz results are obtained using a standard three-sublaminate configuration, in which the facesheets are modeled according to FSDT and the core with a 332 kinematics. The comparison is made against the results reported by Rose et al. (2002) from computations carried out with the finite element code STAGS, in which special-purpose sandwich elements were implemented with the possibility of stacking one or four elements along the thickness direction. Starting from low $t_{c} / a$ ratios (thin cores), an initial increase of the core thickness increases the bending stiffness of the panel and, hence, the global buckling loads are progressively higher in the leftmost part of the curves. At a threshold value of the $t_{c} / a$ ratio, which is different for the thin- and thick-face configurations, the transition to a local buckling instability is observed, and the local buckling load is progressively reduced by further increase of the core thickness. The Ritz solution correctly captures this trend, with buckling loads that are in close proximity with those obtained by the four-layer models in STAGS in both the global and local buckling regime. From Fig. 13, it can be noted that STAGS models using only a single though-the-thickness element tend to underpredict the buckling load. These conclusions hold for both the thin- and thick-face configurations. It is worth highlighting that the elastic properties of the core trigger local buckling modes with a 


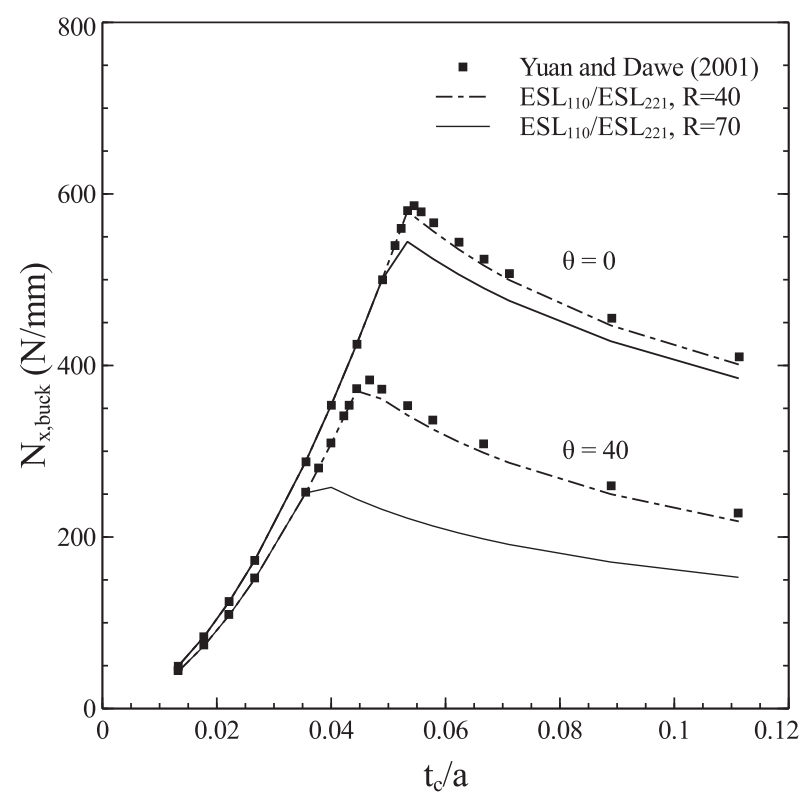

Fig. 14. Transition from global to local buckling response: composite sandwich plate with honeycomb core.

symmetric wrinkling pattern, in which the transverse shear energy stored by the core is much smaller than the transverse extensional energy. A core theory 332 is hence sufficient to grasp the response with a high degree of accuracy.

A last example is addressed that is taken from Yuan and Dawe (2001b), in which a square panel of edge length $225 \mathrm{~mm}$ is considered under the same boundary and loading conditions of the previous case study. Each facesheet is made of one single ply of the orthotropic material $\mathrm{F} 3$, oriented at $0^{\circ}$ or $40^{\circ}$, while the honeycomb core has the elastic properties labeled $\mathrm{C} 11$ in Table 2. Since the in-plane moduli of the honeycomb core are null, a low-order theory can be adopted for the wrinkling analysis. The present results are thus obtained with the sandwich model $\mathrm{ESL}_{110} / \mathrm{ESL}_{221}$. This choice is in agreement with the considerations made before, and has been corroborated by a convergence analysis not reported here for the sake of brevity. Furthermore, the chosen model corresponds with that adopted in Yuan and Dawe (2001a,b), whose results are taken here as reference.

The evolution of the buckling loads with respect to the $t_{c} / a$ ratio is reported in Fig. 14. The comparison with SGUFRitz calculations reveals close matching if the expansion is based upon 40 terms along both directions. However, further spatial refinement is needed to properly capture the highly localized modes characterizing the local instabilities of the configurations investigated here. In particular, it was found that convergence can be reached by enriching the Ritz basis up to 70 terms, leading to the results reported in Fig. 14 with continuous lines. The effect of increasing the number of functions is twofold: firstly the buckling loads in the wrinkling regime are lowered; secondly, the transition from overall to local buckling is anticipated. It is worth noting that the off-axis configuration with $\theta$ oriented at $40^{\circ}$ renders this aspect particularly severe due to the more complex pattern induced by the facesheet anisotropy. With this regard, the proposed approach seems a particularly efficient strategy, as a relatively high number of Ritz functions can be easily accounted for.

\section{Conclusions}

The work has presented a computational approach for analyzing the global and local buckling response of sandwich panels, based on the method of Ritz. The underlying kinematic theory relies upon the subdivision of the structure into sublaminates, and allows to define multiple theories within the same unified framework. Extensive numerical computations have been conducted that focus principally on the wrinkling response of sandwich panels made of orthotropic cores and anisotropic facesheets and subjected to multi-axial loading conditions.

The SGUF variable kinematics approach is particularly attractive inasmuch as the refinement of the theory to be used for the core may need to be tuned depending on the problem under investigation, while keeping a simple theory for the relative thin facesheets. In particular, the results demonstrate that high-order core models, - even with transverse and in-plane displacements representation of order higher then the second and third, respectively - are generally needed in the presence of quasi-isotropic cores undergoing antisymmetric wrinkling. Furthermore, compression loads acting along the weakest inplane direction tend to determine shorter half-wavelengths and, hence, increased requirements for the refinement along the thickness direction. On the contrary, low-order core models suffice whenever the buckling mode is symmetric, thus involving principally the through-thickness normal strain with a negligibly small amount of transverse shear. When compared to 
numerical techniques developed in the past and based on pre-defined kinematic models, the proposed approach offers a broader range of use and allows maximizing the ratio between accuracy and number of degrees of freedom.

A further advantage of the proposed approach is provided by the efficiency of the proposed Ritz implementation, which is based on the use of Legendre polynomials. Accurate results can be derived with a small computational effort, while guaranteeing FEM-like accuracy, as demonstrated by the comparison against numerical results. Furthermore, the efficiency of the basis can be exploited to predict buckled configurations with long and short half-wavelengths, thus allowing to capture the transitions between global and local modes within the same unified framework.

The comparison against closed-form results demonstrates good matching in the case of axial loads, while discrepancies can be relevant whenever multi-axial conditions are of concern or, more generally, when the buckling mode does not verify some assumptions upon which the closed-form solution relies. In contrast with the strength of material approach of common design formulae, the present approach considers the wrinkling response at panel level. Nevertheless, it is believed that it can be successfully employed to help reducing conservativeness, especially in those cases characterized the presence of multi-axial loads.

For the above reasons the SGUF-Ritz approach appears as a suitable tool for the preliminary and intermediate design phases, where thousands of configurations need to be analyzed or sensitivity studies conducted. Further research work will be directed toward the extension of the present approach to account for the effects of initial imperfections, with the aim of deriving improved knock-down factors to be used in conjunction with linearized buckling predictions.

\section{Declarations of interests}

None.

\section{References}

Allen, H. (1969). Analysis and design of structural sandwich panels. Oxford, UK: Pergamon Press.

Babu, C., \& Kant, T. (1999). Two shear deformable finite element models for buckling analysis of skew fibre-reinforced composite and sandwich panels.

Composite Structures, 46, 115-124.

Babu, C., \& Kant, T. (20 0 0). Refined higher order finite element models for thermal buckling of laminated composite and sandwich plates.

Journal of Thermal

Stresses, 23, 111-130.

Bazant, Z., \& Beghini, A. (2006). Stability and finite strain of homogenized structures soft in shear: Sandwich or fiber composites, and layered bodies

International Journal of Solids and Structures, 43, 1571-1593.

Berdichevsky, V. (1979). Variational-asymptotic method of constructing a theory of shells: Pmm vol. 43, no. 4, 1979, pp. 664-687. Journal of Applied Mathematics and Mechanics, 43, 711-736.

Berdichevsky, V. (2010). An asymptotic theory of sandwich plates. International Journal of Engineering Science, 48, 383-404.

Birman, V., \& Bert, C. (2004). Wrinkling of composite-facing sandwich panels under biaxial loading. Journal of Sandwich Structures E Materials, 6, $217-237$.

Birman, V., \& Kardomateas, G. (2018). Review of current trends in research and applications of sandwich structures. Composites Part B: Engineering, 142, $221-240$.

Carlsson, L., \& Kardomateas, G. (2011). Structural and failure mechanics of sandwich composites. The Netherlands: Springer.

Carrera, E. (2002). Theories and finite elements for multilayered, anisotropic, composite plates and shells. Archives of Computational Methods in Engineering, 9, 87-140.

Carrera, E. (2003a). Historical review of zig-zag theories for multilayered plates and shells. Applied Mechanics Reviews, 56, $287-308$.

Carrera, E. (2003b). Theories and finite elements for multilayered plates and shells: A unified compact formulation with numerical assessment and benchmarking. Archives of Computational Methods in Engineering, 10, 215-296.

Carrera, E., Pagani, A., \& Valvano, S. (2017). Shell elements with through-the-thickness variable kinematics for the analysis of laminated composite and sandwich structures. Composites Part B: Engineering, 111, 294-314.

Cho, Y., \& Averill, R. (2000). First-order zig-zag sublaminate plate theory and finite element model for laminated composite and sandwich panels. Composite Structures, 50, 1-15.

Coburn, B., \& Weaver, P. (2016). Buckling analysis, design and optimisation of variable-stiffness sandwich panels. International Journal of Solids and Structures, 96, 217-228.

Demasi, L. (2009a). $\infty^{6}$ Mixed plate theories based on the generalized unified formulation. Part I: Governing equations. Composite Structures, 87, 1.

Demasi, L. (2009b). $\infty^{6}$ Mixed plate theories based on the generalized unified formulation. Part II: Layerwise theories. Composite Structures, 87 , 12-22.

Demasi, L. (2009c). $\infty^{6}$ Mixed plate theories based on the generalized unified formulation. part III: Advanced mixed high order shear deformation theories. Composite Structures, $87,83-194$

Demasi, L., \& Yu, W. (2013). Assess the accuracy of the variational asymptotic plate and shell analysis using the generalized unified formulation. Mechanics of Advanced Materials and Structures, 20, 227-241.

D’Ottavio, M. (2016). A sublaminate generalized unified formulation for the analysis of composite structures. Composite Structures, 142, $187-199$.

D’Ottavio, M., Dozio, L., Vescovini, R., \& Polit, O. (2016). Bending analysis of composite laminated and sandwich structures using sublaminate variable-kinematic ritz models. Composite Structures, 155, 45-62.

D'Ottavio, M., \& Polit, O. (2015). Linearized global and local buckling analysis of sandwich struts with a refined quasi-3D model. Acta Mechanica, 226, $81-101$.

D’Ottavio, M., Polit, O., Ji, W., \& Waas, A. (2016). Benchmark solutions and assessment of variable kinematics models for global and local buckling of sandwich struts. Composite Structures, 156, 125-134.

Dozio, L., \& Carrera, E. (2012). Ritz analysis of vibrating rectangular and skew multilayered plates based on advanced variable-kinematic models. Composite Structures, 94, 2118-2128.

Fagerberg, L. (2003). The effect of local bending stiffness on the wrinkling of sandwich panels. Proceedings of the Institution of Mechanical Engineers, Part M: Journal of Engineering for the Maritime Environment, 217, 111-119. 
Fagerberg, L., \& Zenkert, D. (2005a). Effects of anisotropy and multiaxial loading on the wrinkling of sandwich panels. Journal of Sandwich Structures E Materials, 7, 177-194.

Fagerberg, L., \& Zenkert, D. (2005b). Imperfection-induced wrinkling material failure in sandwich panels. Journal of Sandwich Structures E' Materials, 7, 195-219.

Fazzolari, F., \& Carrera, E. (2013). Thermo-mechanical buckling analysis of anisotropic multilayered composite and sandwich plates by using refined variable-kinematics theories. Journal of Thermal Stresses, 36, 321-350.

Frostig, Y., Baruch, M., Vilnay, O., \& Sheinman, I. (1992). High-order theory for sandwich-beam behavior with transversely flexible core. Journal of Engineering Mechanics, 118, 1026-1043.

Frostig, Y., \& Thomsen, O. (2011). Non-linear thermo-mechanical behaviour of delaminated curved sandwich panels with a compliant core. International Journal of Solids and Structures, 48, 2218-2237.

Hadi, B., \& Matthews, F. (1998). Predicting the buckling load of anisotropic sandwich panels: An approach including shear deformation of the faces. Composite Structures, 42, 245-255.

Hoff, N. (1945). The buckling of sandwich-type panels. Journal of the Aeronautical Sciences, 12, 285-297.

Ji, W., \& Waas, A. (2007). Global and local buckling of a sandwich beam. Journal of Engineering Mechanics, 133, $230-237$.

Ji, W., \& Waas, A. (2008). Wrinkling and edge buckling in orthotropic sandwich beams. Journal of Engineering Mechanics, 134, 455-461.

Ji, W., \& Waas, A. (2012). Accurate buckling load calculations of a thick orthotropic sandwich panel. Composites Science and Technology, 72, 1134-1139.

Jones, R. (1998). Mechanics of composite materials. CRC Press.

Jones, R. (2006). Buckling of bars, plates, and shells. Blacksburg, VA: Bull Ridge Corporation.

Kardomateas, G. (2005). Wrinkling of wide sandwich panels/beams with orthotropic phases by an elasticity approach. Journal of Applied Mechanics, 72, $818-825$.

Kardomateas, G. (2010). An elasticity solution for the global buckling of sandwich beams/wide panels with orthotropic phases. Journal of Applied Mechanics, $77,021015$.

Khalili, S., \& Malekzadeh, F. K. (2015). Buckling analysis of composite sandwich plates with flexible core using improved high-order theory. Mechanics of Advanced Materials and Structures, 22, 233-247.

Khdeir, A., Reddy, J., \& Frederick, D. (1989). A study of bending, vibration and buckling of cross-ply circular cylindrical shells with various shell theories. International Journal of Engineering Science, 27, 1337-1351.

Kim, C., \& Hong, C. (1988). Buckling of unbalanced anisotropic sandwich plates with finite bonding stiffness. AIAA Journal, $26,982-988$.

Koissin, V., Shipsha, A., \& Skvortsov, V. (2011). Wrinkling in sandwich panels - An analytical approach. Journal of Sandwich Structures E Materials, 13, 705-730.

Léotoing, L., Drapier, S., \& Vautrin, A. (2002). First applications of a novel unified model for global and local buckling of sandwich columns. European Journal of Mechanics - A/Solids, 21, 683-701.

Ley, R., Lin, W., \& Mbanefo, U. (1999). Facesheet wrinkling in sandwich structures. Technical Report, CR-1999-208994, NASA.

Librescu, L., \& Reddy, J. (1989). A few remarks concerning several refined theories of anisotropic composite laminated plates. International Journal of Engineering Science, 27, 515-527.

Lopatin, A., \& Morozov, E. (2008). Symmetrical facing wrinkling of composite sandwich panels. Journal of Sandwich Structures E Materials, $10,475-497$.

Niu, K., \& Talreja, R. (1999). Modeling of wrinkling in sandwich panels under compression. Journal of Engineering Mechanics, 125 , 875-883.

Noor, A., Burton, W., \& Bert, C. (1996). Computational models for sandwich panels and shells. Applied Mechanics Review, 49, $155-199$.

Pandit, M., Singh, B., \& Sheikh, A. (2008). Buckling of laminated sandwich plates with soft core based on an improved higher order zigzag theory. Thin-Walled Structures, 46, 1183-1191.

Phan, C., Bailey, N., Kardomateas, G., \& Battley, M. (2012). Wrinkling of sandwich wide panels/beams based on the extended high-order sandwich panel theory: Formulation, comparison with elasticity and experiments. Archive of Applied Mechanics, 82, 1585-1599.

Phan, C., Frostig, Y., \& Kardomateas, G. (2012). Analysis of sandwich beams with a compliant core and with in-plane rigidity extended high-order sandwich panel theory versus elasticity. Journal of Applied Mechanics, 79, 041001.

Phan, C., Frostig, Y., \& Kardomateas, G. (2013). Free vibration of unidirectional sandwich panels, part II: Incompressible core. Journal of Sandwich Structures E' Materials, 15, 412-428.

Phan, C., Kardomateas, G., \& Frostig, Y. (2012). Global buckling of sandwich beams based on the extended high-order theory. AIAA Journal, 50, $1707-1716$.

Piskunov, V., Verijenko, V., Adal, S., \& Summers, E. (1993). A higher-order theory for the analysis of laminated plates and shells with shear and normal deformation. International Journal of Engineering Science, 31, 967-988.

Plantema, F. (1966). Sandwich construction: The bending and buckling of sandwich beams, plates and shells. New York, USA: John Wiley and Sons.

Rao, K. (1985). Buckling analysis of anisotropic sandwich plates faced with fiber-reinforced plastics. AIAA Journal, $23,1247-1253$.

Reddy, J. (2004). Mechanics of laminated composite plates and shells: theory and analysis. Boca Raton: CRC Press.

Reddy, J., \& Liu, C. (1985). A higher-order shear deformation theory of laminated elastic shells. International Journal of Engineering Science, 23, 319-330.

Rose, C., Moore, D., Knight, N., \& Rankin, C. (2002). Finite element modeling of the buckling response of sandwich panels. In Proceedings of the 43rd AIAA/ASME/ASCE/AHS/ASC structures, structural dynamics, and materials conference, Denver, CO. AIAA-2002-1517.

Stone, M., \& Chandler, H. (1996). Errors in double sine series solutions for simply supported symmetrically laminated plates. International Journal of Mechanical Sciences, 38, 517-526.

Suzuki, T., Aoki, T., Ogasawara, T., \& Fujita, K. (2017). Nonablative lightweight thermal protection system for mars aeroflyby sample collection mission. Acta Astronautica, 136, 407-420.

Vescovini, R., \& Dozio, L. (2016). A variable-kinematic model for variable stiffness plates: Vibration and buckling analysis. Composite Structures, 142, 15-26.

Vescovini, R., Dozio, L., D'Ottavio, M., \& Polit, O. (2018). On the application of the Ritz method to free vibration and buckling analysis of highly anisotropic plates. Composite Structures, 192, 460-474.

Vinson, J. (1999). The behavior of sandwich structures of isotropic and composite materials. Lancaster, PA: Technomic.

Vonach, W., \& Rammerstorfer, F. (2000). Wrinkling of thick orthotropic sandwich plates under general loading conditions. Archive of Applied Mechanics, 70, $338-348$.

Wu, Z., Raju, G., \& Weaver, P. (2012). Comparison of variational, differential quadrature, and approximate closed-form solution methods for buckling of highly flexurally anisotropic laminates. Journal of Engineering Mechanics, 139, 1073-1083.

Yu, K., Hu, H., Tang, H., Giunta, G., Potier-Ferry, M., \& Belouettar, S. (2015). A novel two-dimensional finite element to study the instability phenomena of sandwich plates. Computer Methods in Applied Mechanics and Engineering, 283, 1117-1137.

Yu, W., Hodges, D., \& Volovoi, V. (2002). Asymptotic construction of reissner-like composite plate theory with accurate strain recovery. International Journal of Solids and Structures, 39, 5185-5203.

Yuan, W., \& Dawe, D. (2001a). Overall and local buckling of sandwich plates with laminated faceplates, part I: Analysis. Computer Methods in Applied Mechanics and Engineering, 190, 5197-5213.

Yuan, W., \& Dawe, D. (2001b). Overall and local buckling of sandwich plates with laminated faceplates, part II: Applications. Computer Methods in Applied Mechanics and Engineering, 190, 5215-5231.

Yuan, Z., Kardomateas, G., \& Frostig, Y. (2015). Finite element formulation based on the extended high-order sandwich panel theory. AIAA Journal, 53, 3006-3015. 